\title{
1 Selective amide bond formation in redox-active coacervate protocells
}

\author{
Jiahua Wang, ${ }^{[\mathrm{a}]}$ Manzar Abbas, ${ }^{[\mathrm{a}]}{ }^{\mathrm{Junyou}}$ Wang ${ }^{[\mathrm{b}]}$ and Evan Spruijt ${ }^{*}[\mathrm{a}]$
}

3

$4{ }^{[a]}$ Radboud University, Institute for Molecules and Materials, Heyendaalseweg 135, 6525 AJ Nijmegen, the Netherlands

$5{ }^{[b]}$ State Key Laboratory of Chemical Engineering and Shanghai Key Laboratory of Multiphase Materials Chemical

6 Engineering, East China University of Science and Technology, Shanghai 200237, China

$7 \quad$ *Email: e.spruijt@science.ru.nl, junyouwang@ecust.edu.cn

\section{Abstract}

10 Membraneless compartments, like complex coacervates droplets, are promising protocell models

11 because of their ability to sequester a wide range of guest molecules and their catalytic properties.

12 However, it remains unclear how the building blocks of life, including peptides, could be

13 synthesized from primitive precursor molecules inside such protocells. Here, we develop a new

14 protocell model formed by phase separation of prebiotically relevant small redox-active

15 ferricyanide $\left(\mathrm{Fe}(\mathrm{CN}) 6^{3-}\right)$ /ferrocyanide $\left(\mathrm{Fe}(\mathrm{CN}) 6^{4-}\right)$ molecules and a cationic peptide. The assembly

16 of these coacervate protocells can be regulated by redox chemistry and they act as oxidizing hubs

17 for sequestered metabolites, such as $\mathrm{NAD}(\mathrm{P}) \mathrm{H}$, and fiber precursors. Interestingly, we show that

18 the oxidizing potential of ferricyanide inside coacervates can be harnessed to drive the selective

19 formation of amide bonds between prebiotically relevant amino thioacids and amino acids or

20 peptides. We demonstrate that aminoacylation is enhanced in $\mathrm{Fe}(\mathrm{CN})_{6}^{3-} /$ peptide coacervate

21 dispersions compared to the surrounding dilute phase, and selective for amino acids that interact

22 less strongly with the coacervates. We finally use this amide bond formation to create self- 
23 reinforcing coacervates by reacting hydrophobic amino thioacids to amines on the protocell

24 scaffold and show that this significantly enhances their salt resistance. These results provide an

25 important step towards the prebiotically relevant integration of redox chemistry in cell-like

26 compartments.

29 Introduction

30 Amide bond formation is an essential chemical reaction in all forms of life that is catalyzed by

31 highly evolved biomolecular machinery. However, before ribosomes and specialized enzymes

32 became capable of protein synthesis, ${ }^{1,2}$ alternative, simple prebiotic routes to create peptide bonds

33 in a spatiotemporally controlled way likely existed. Protocellular compartments provide a

34 promising platform to localize chemical reactions relevant to life. ${ }^{3-5}$ What the nature of such

35 protocellular compartments capable of peptide synthesis could be, remains unknown. As plausible

36 precursors to peptides, $\alpha$-aminothioacids (AA-SH) ${ }^{6}$ and acetylated aminothioacids (Ac-AA-SH) ${ }^{7}$

37 have been shown to be formed in aqueous conditions at near-neutral $\mathrm{pH}$. They are considered

38 interesting alternatives to biological thioesters for prebiotic peptide ligation, and can be ligated into

39 peptide with the aid of an oxidizing catalyst, such as ferricyanide. ${ }^{7-10}$ However, high concentrations

40 of reactants and catalysts are typically required for these oxidative peptide ligations, which may

41 not have been easy to reach. Recent work has demonstrated that some micro-compartments and

42 protocell systems could compartmentalize (bio)chemical reactions, and act as catalytic

43 microreactors or reaction localization centers with potential for prebiotic peptide ligation. ${ }^{11-16}$

44 In particular, membraneless compartments based on complex coacervates have been

45 considered as versatile protocell models in the origins of life research. ${ }^{5,16-19}$ Coacervate droplets 
46 are formed spontaneously by liquid-liquid phase separation, resulting in a polymer- or peptide-rich,

47 cell-sized dense coacervate phase, and a coexisting dilute phase. These membraneless droplets can

48 easily take up and concentrate guest molecules from their surroundings due to charge

49 complementarity or hydrophobicity. ${ }^{16,20,21}$ The ability to up-concentrate and exchange guests make

50 the coacervate droplets capable of supporting biochemical reactions, and sometimes enhancing the

51 activity of catalysts, such as ribozymes, ${ }^{12,13}$ or enzymes in cascade reactions, ${ }^{15,22}$ thereby increasing

52 the overall rate. Recent work has demonstrated that coacervate droplets can be active, ${ }^{18,23}$ and that

53 their formation and dissolution can be regulated by environmental changes, such as $\mathrm{pH},{ }^{24,25}$

54 temperature, ${ }^{26,27}$ light, ${ }^{28}$ enzymatic reactions, ${ }^{23,29-31}$ or fuel-driven chemical reaction cycles. ${ }^{18}$

55 To date, research on coacervates as reaction localization centers has mostly focused on

56 enzymatic reactions, or reactions involving complex RNAzymes (ribozymes). However, the

57 fundamental biochemical reactions that lead to the formation of peptides, which themselves are

58 often the building blocks of coacervates, have not been studied. For coacervates to be a plausible

59 protocell model, they must be able to support a prebiotically relevant ligation reaction of peptides,

60 and ultimately, link this reaction to the construction of new coacervates. Here, we show that

61 ferricyanide-containing coacervates can potentially fulfill this role. These coacervates can be used

62 to oxidize not only a variety of common metabolites, but also amino thioacids, which can

63 subsequently be aminoacylated yielding a product with a new amide bond.

64 Coacervates with ferrocyanide, the reduced form of ferricyanide, as multivalent anion have

65 already been reported by Bungenberg-de Jong and Kruyt in $1929 .{ }^{32}$ They used gelatin at low $\mathrm{pH}$

66 as a long polycation and observed small punctated droplets upon mixing with potassium

67 ferrocyanide. However, the redox potential of the iron centers inside these coacervates remains

68 unclear. More recently, it was shown that coacervates can be formed from much smaller cationic

69 oligopeptides, such as oligolysine and oligoarginine with as little as five amino acids, complexed 
70 with either small tri- or tetravalent anions, like ADP and ATP. ${ }^{19}$ We therefore hypothesized that it

71 should be possible to make coacervates of the redox couple ferri- and ferrocyanide with a cationic

72 polypeptide in such a way that ferrocyanide, the reduced state with a 4- charge, would form stable

73 droplets, but ferricyanide, the oxidized state with a 3- charge, would not. This would enable the

74 selective compartmentalization of guest molecules only under certain redox potential in the

75 environment. In addition, from an origins of life perspective, the environment on early Earth was

76 likely depleted of oxygen, a strong oxidizing agent that is widely used in living systems, for a

77 considerable period. Alternative oxidizing agents, such as ferricyanide $\left(\mathrm{Fe}(\mathrm{CN}){ }^{3-}\right)$, could have has

78 been essential in the prebiotic activation of building blocks for peptide ligation, ${ }^{7,9,33,34}$ and oxidation

79 of metabolites. ${ }^{35}$ By condensing the ferricyanide with an oppositely charged peptide at low ionic

80 strength, it may be possible to localize these reactions in a droplet compartment and enrich the

81 coacervate with the products of ferricyanide-catalyzed oxidation reactions.

82 Here, we show that both ferricyanide and ferrocyanide ions can be condensed into coacervate

83 droplets with short cationic polypeptides depending on the ionic strength. These droplets are

84 responsive and can be regulated by redox chemistry. They act as prebiotic oxidizing hubs for

85 metabolites and common electron donors, such as NADH, NADPH and GSH, thus taking the role

86 of oxygen as terminal electron acceptor. Besides small metabolites, aromatic thiols such as benzoyl

87 cysteine can be oxidized inside ferricyanide-based coacervates, leading to their assembly into

88 stacked filaments, which further bundle into rigid fibers that resemble a cytoskeletal network inside

89 and around the protocells. Moreover, we show that the oxidizing potential of ferricyanide-based

90 coacervates can be harnessed to drive the formation of peptide bonds between amino acid and

91 amino thioacids which are considered as potential prebiotic precursors of amino acids. We

92 demonstrate that amino thioacid ligation is enhanced in $\mathrm{Fe}(\mathrm{CN})_{6}{ }^{3-} /$ peptide coacervate dispersions

93 compared to the surrounding dilute phase due to the local high ferricyanide concentration. The 
94 coacervate environment imposes a selection pressure that results in kinetic pathway selection and

95 a strong, preferential incorporation of certain amino acids. When amino acids or short peptides are

96 used as client substrates, the reaction products can leave the coacervate droplets again, but they can

97 also be anchored to the coacervate compartments by using peptides containing amine groups as

98 coacervating scaffolds. We show that this strategy can be used to create self-reinforcing

99 coacervates, in which hydrophobic amino acid residues are ligated to the coacervate building

100 blocks and enhance their stability. In short, our results show that prebiotically relevant ferricyanide-

101 based coacervate protocells are versatile oxidizing hubs that exist in aqueous solution, in which

102 metabolites can be converted, fibrous networks assembled and peptides synthetized.

\section{Results and discussion}

\section{Coacervation of ferricyanide/ferrocyanide and polypeptides}

106 Small, multivalent ions can be condensed into liquid coacervate droplets by complexation with an

107 oppositely charged peptide or polymer. ${ }^{5}$ We sought to use this principle to create coacervate 108 protocells that can concentrate a prebiotically relevant redox catalyst to enable localized peptide

109 synthesis. Ferricyanide is a trivalent anion and its reduced form, the tetravalent ferrocyanide, has

110 been shown to be able to form coacervates with gelatin at low $\mathrm{pH} .{ }^{32} \mathrm{We}$ used cationic peptides to

111 induce phase separation of ferri- and ferrocyanide. Spherical complex (heterotypic) coacervate

112 droplets were readily formed as a turbid dispersion via spontaneous liquid-liquid phase separation

113 associated with the charge neutralization of a range of peptides $\left((\mathrm{Lys})_{10,}(\mathrm{Lys})_{20},\left(\mathrm{Lys}(\mathrm{Me})_{3}\right)_{20}\right.$,

114 (Lys)30, $\left(\operatorname{Lys}(\mathrm{Me})_{3}\right)_{30}$, poly-L-lysine (pLys) and $\left.(\mathrm{Arg})_{10}\right)$ in the presence of ferrocyanide or

115 ferricyanide (Fig. 1a, Supplementary Fig. 4). (Arg) 10 formed coacervates with both ferrocyanide

116 and ferricyanide, while (Lys) $10 /(\text { Lys })_{20}$ only formed coacervates with tetravalent ferrocyanide in 
117 salt-free solution. The difference between (Lys) $)_{10}$ and (Arg) 10 can be explained by the higher $\mathrm{p} K_{\mathrm{a}}$

118 of the basic residue in arginine compared to lysine, which may generate more stable ionic

119 interactions at a given chain length. ${ }^{36}$ Increasing the length of polylysine to (Lys) $)_{30}$ resulted in the

120 formation of coacervates with trivalent ferricyanide as well (Supplementary Fig. 4c), in agreement

121 with previous studies involving nucleotides. ${ }^{19,27}$

122

a
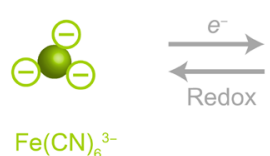

$\mathrm{Fe}(\mathrm{CN})_{6}{ }^{3}$

$\mathrm{Fe}(\mathrm{CN})_{6}{ }^{4-}$

\section{b}
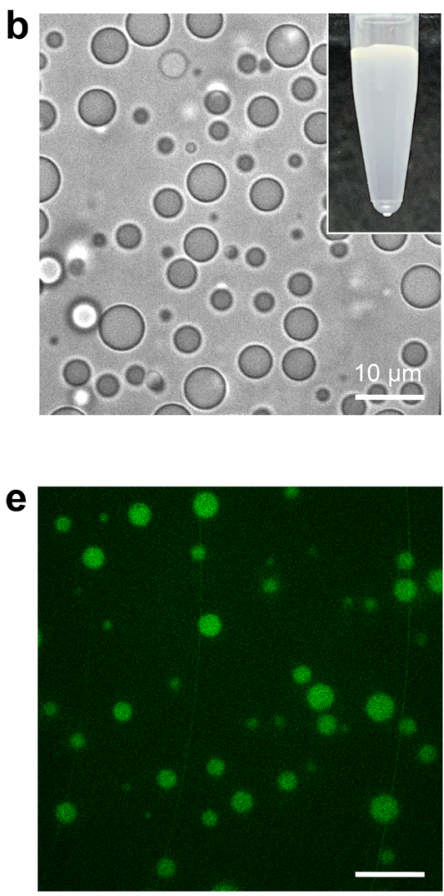

C

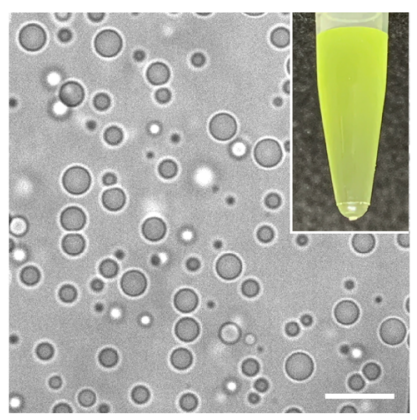

f

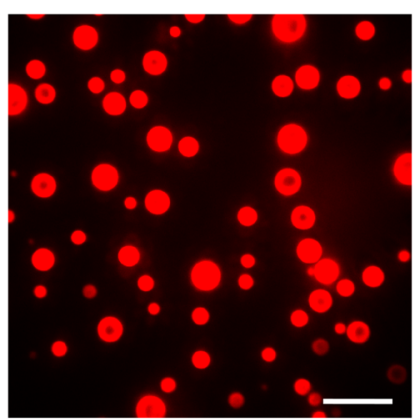

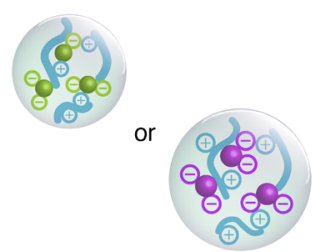

Coacervates

d
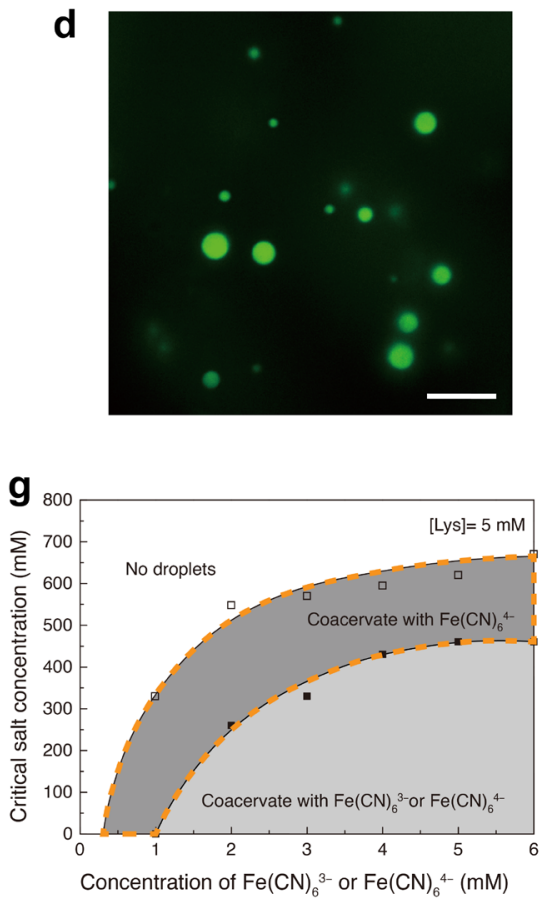

Figure 1. (a) Schematic illustration of associative liquid-liquid phase separation of $\mathrm{Fe}(\mathrm{CN})_{6}{ }^{3-} / \mathrm{Fe}(\mathrm{CN})_{6}{ }^{4-}$ and plys to produce coacervate droplets. (b) Optical microscope images of $\mathrm{Fe}(\mathrm{CN})_{6}{ }^{4-} / \mathrm{pLys}$ droplets prepared at $1 \mathrm{mM} \mathrm{Fe}(\mathrm{CN})_{6}{ }^{4-}$ and $5 \mathrm{mM}$ pLys (monomer basis), and (c) at $2 \mathrm{mM} \mathrm{Fe}(\mathrm{CN})_{6}{ }^{3-}$ and $5 \mathrm{mM}$ pLys (monomer basis). Insets show photographs of the corresponding turbid suspensions. (d-f) Fluorescence microscopy images of $\mathrm{Fe}(\mathrm{CN})_{6}{ }^{4-} / \mathrm{pLys}$ droplets with various client molecules: (d) pyranine, (e) NADPH, (f) poly-rU 15 (scale bars indicate $10 \mu \mathrm{m}$ ). (g) Critical salt concentration of $\mathrm{Fe}(\mathrm{CN})_{6}{ }^{3-}$ and $\mathrm{Fe}(\mathrm{CN})_{6}{ }^{4-}$ coacervates with a fixed concentration of $5 \mathrm{mM}$ pLys, determined from turbidity titrations. 
131 The obtained coacervates with a diameter of about 2 to $3 \mu \mathrm{m}$ were observed in a PLL-g-PEG

132 functionalized microchamber (Figure 1b-c). These microdroplets exhibited liquid-like properties:

133 they wetted the bottom glass surface and sedimenting coacervates coalesced with coacervates

134 already present at the bottom of the microchambers to form bigger droplets. The droplets 135 sequestered negatively charged client solutes, including pyranine, $\mathrm{NAD}(\mathrm{P}) \mathrm{H}$, and ribonucleic acids

136 (Figure 1d-f, Supplementary Fig. 4). Determination of the partitioning of ferricyanide and

137 ferrocyanide by Uv-vis spectroscopy (see Methods) showed that both these multivalent scaffolding

138 anions were highly concentrated in the coacervate droplets: the internal concentration in coacervate

139 droplets was 100 times higher than the surrounding dilute aqueous phase. For example, for samples

140 prepared from $2 \mathrm{mM}$ ferricyanide and pLys (5mM lysine monomers) solutions, we found an

141 internal ferricyanide concentration of $\sim 30 \mathrm{mM}$, compared to $\sim 0.3 \mathrm{mM}$ in the surrounding aqueous

142 phase. (Supplementary Fig. 5). Surprisingly, the concentration ratio for ferri- and ferrocyanide was

143 the same, despite their different valency. We attribute this to the formation of a tighter complex

144 between ferricyanide and polycations, which results in a more hydrophobic coacervate, ${ }^{27}$ an effect

145 that has previously been observed with ferricyanide in polyelectrolyte brushes. ${ }^{37}$ A similar 100x

146 concentration was previously found for a negatively charged bis-carboxylic acid ligand, which can

147 exist in a tetravalent (4-) ring configuration, in coacervates with cationic polymers. ${ }^{38}$ These initial

148 observations suggest that the spontaneous assembly of peptides and ferricyanide or ferrocyanide

149 could be developed as potential prebiotic membraneless compartments.

150 Typically, coacervates formed by charge-charge interaction are sensitive to the ionic strength

151 and they exhibit a critical salt concentration (CSC) above which phase separation does not take

152 place. Since ferricyanide and ferrocyanide have a different net charge, their CSC is likely different,

153 even though the previously discussed hydration differences may decrease that effect. A difference

154 in CSC would allow for selective compartmentalization controlled by redox chemistry. To 
155 determine the conditions under which the redox couple ferri- and ferrocyanide could give rise to

156 reversible coacervate formation and dissolution, we evaluated the salt resistance of $\mathrm{Fe}(\mathrm{CN})_{6}{ }^{4-}$ and

$157 \mathrm{Fe}(\mathrm{CN})_{6}{ }^{3-}$-based coacervates. As a model peptide, we focused on pLys $\left(M_{\mathrm{w}}=15-30 \mathrm{kDa}\right)$.

158 Supplementary Fig. 6 shows turbidity-based titration curves of pLys (5 mM monomer units), as a

159 function of $\mathrm{Fe}(\mathrm{CN})_{6}{ }^{4-}$ or $\mathrm{Fe}(\mathrm{CN})_{6}{ }^{3-}$, and as a function of salt concentration. From plots of the

160 turbidity we determined the critical salt concentration (CSC), the point at which coacervate droplets

161 completely disappear. Figure $1 \mathrm{~g}$ shows the resulting phase diagram of both $\mathrm{Fe}(\mathrm{CN})_{6}{ }^{4-}$ and

$162 \mathrm{Fe}(\mathrm{CN})_{6}{ }^{3-}$ coacervates. As expected, $\mathrm{Fe}(\mathrm{CN})_{6}{ }^{4-}$-based coacervates have a higher salt resistance,

163 expressed by their $\mathrm{CSC}$, compared to $\mathrm{Fe}(\mathrm{CN})_{6}{ }^{3-}$-based coacervates. Our results are in good

164 agreement with previous studies with nucleotide/pLys-based coacervates, where the ATP/pLys

165 droplets have a higher CSC than ADP/pLys droplets, in line with their valency. ${ }^{19,30}$

\section{Redox chemistry of ferri/ferrocyanide in coacervates}

168 We next exploited the redox activity of the droplets in the critical salt concentration window 169 highlighted in Fig. 1g. To illustrate the feasibility of the redox cycling proposed in Fig. 2a to induce 170 phase separation and droplet dissolution, we prepared mixtures of $\mathrm{Fe}(\mathrm{CN})_{6}{ }^{4-}$ with pLys and $171 \mathrm{Fe}(\mathrm{CN})_{6}^{3-}$ with pLys under identical conditions within the highlighted region of Fig. $1 \mathrm{~g}$ between

172 the two binodal lines. The original $\mathrm{Fe}(\mathrm{CN})_{6}{ }^{4-}$-containing mixtures and $\mathrm{Fe}(\mathrm{CN}) 6^{3-}$-containing 173 mixtures are white turbid and yellowish transparent, respectively. When observed under the 174 microscope, the $\mathrm{Fe}(\mathrm{CN})_{6}{ }^{4-}$-containing mixtures had clearly condensed into droplets, while the $175 \mathrm{Fe}(\mathrm{CN})_{6}{ }^{3-}$-containing mixtures remained a homogeneous solution. Upon oxidation of the $\mathrm{Fe}(\mathrm{CN}) 6^{4-}$ 176 -coacervates by $\mathrm{S}_{2} \mathrm{O}_{8}{ }^{2-}$, the originally white turbid solution turned yellowish transparent, and no 177 droplets could be observed under the microscope (Fig. 2b). Conversely, after reduction of the $178 \mathrm{Fe}(\mathrm{CN})_{6}^{3-}$-containing mixtures with GSH or NADH (Fig. 2b-d), the original, light-yellow 
180 under the microscope.

a

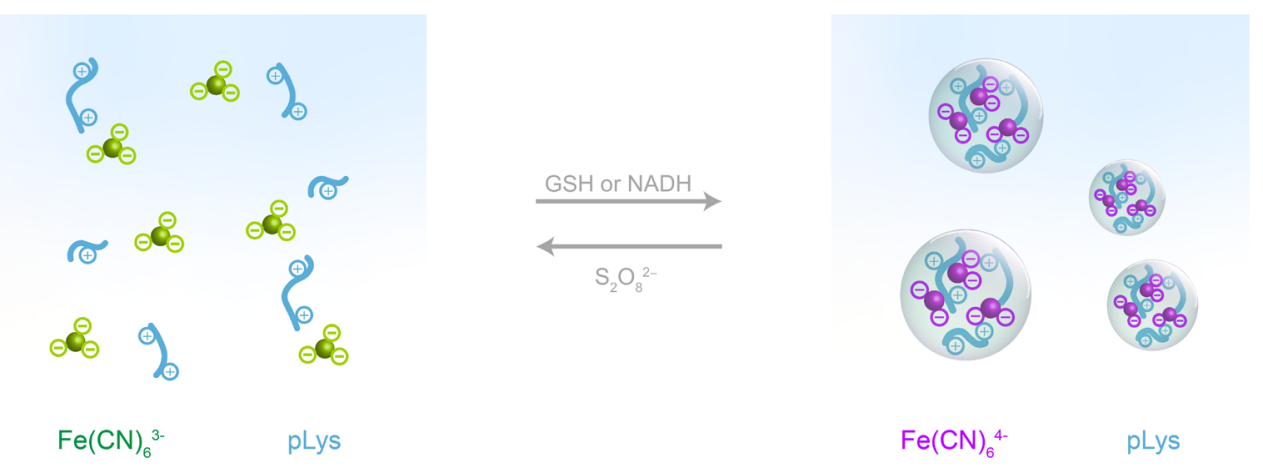

\section{b}
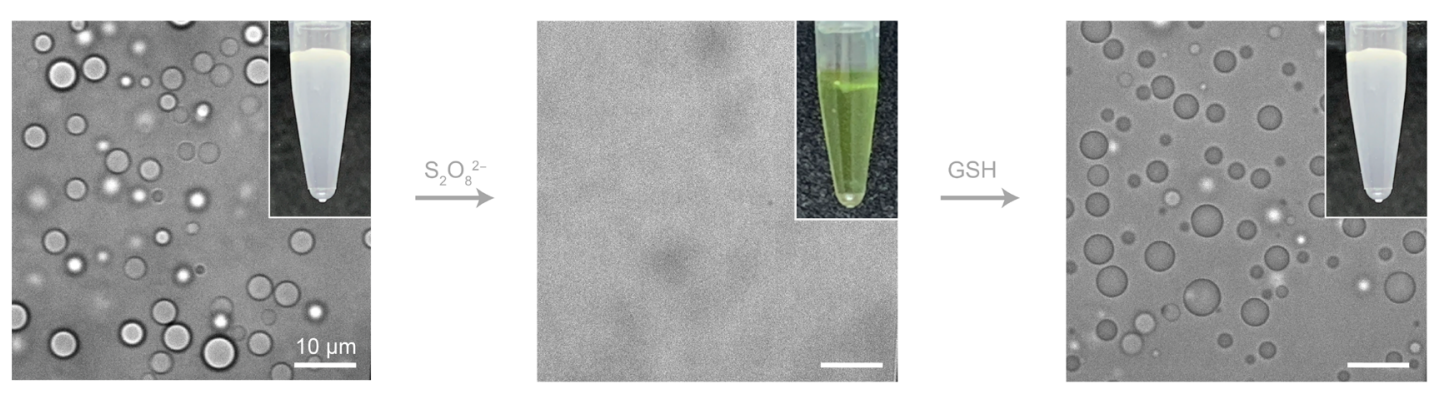

C
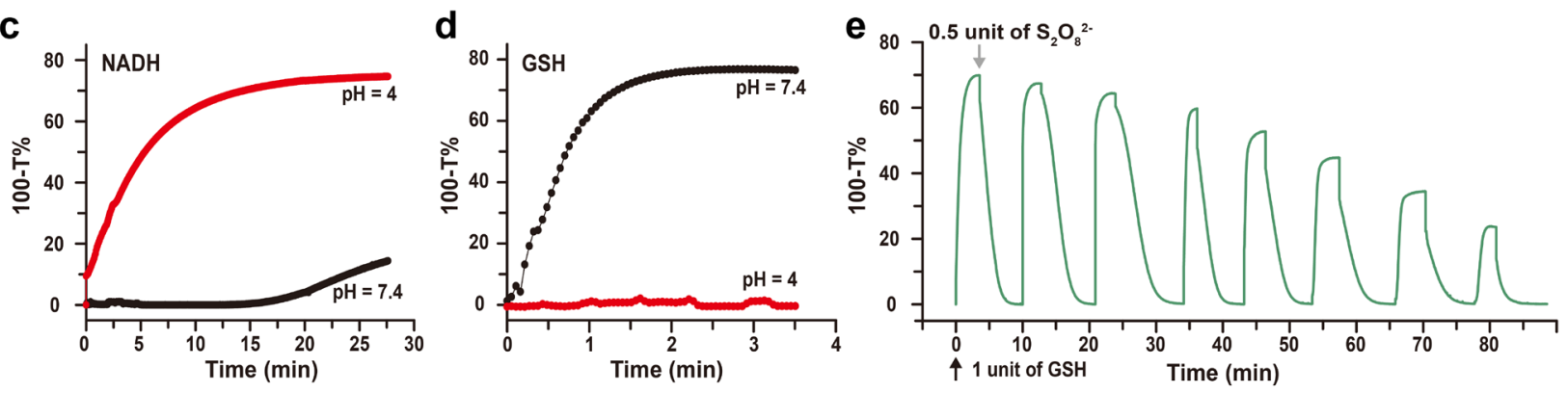

Figure 2. (a) Schematic illustration of the redox reaction network underlying dynamic and reversible formation and dissolution of $\mathrm{Fe}(\mathrm{CN})_{6}{ }^{4-} / \mathrm{pLys}$ coacervate droplets. (b) Optical observation of droplet dissolution by $\mathrm{S}_{2} \mathrm{O}_{8}{ }^{2-}$ addition to $\mathrm{Fe}(\mathrm{CN})_{6}{ }^{4-} / \mathrm{pLys}$ coacervates dispersion and droplet formation by GSH addition. Scale bars: $10 \mu \mathrm{m}$. Insets show droplets induced by addition of 1 equivalent of NADH at $\mathrm{pH} 4$, but not at $\mathrm{pH}$ 7.4. (d) Same as (c), but induced by addition of 1 equivalent of GSH, which proceeds at $\mathrm{pH} 7.4$, but not at $\mathrm{pH} 4$. (e) Alternating additions of GSH and $\mathrm{S}_{2} \mathrm{O}_{8}{ }^{2-}$ at $\mathrm{pH} 7.4$ show that condensation and dissolution are both reversible and that the system can be switched multiple times between a compartmentalized droplet state and a single-phase homogeneous solution. 
The oxidation reaction showed a clear $\mathrm{pH}$ dependence (Fig. 2c,d). We were able to use a

192 stoichiometric amount of GSH to turn a homogeneous ferricyanide solution into a dispersion of 193 ferrocyanide droplets in the presence of pLys within 5 minutes in neutral conditions (pH 7.4, Fig.

194 2b,d), while no conversion occurred under acidic conditions ( $\mathrm{pH} 4)$. When using NADH as 195 reducing agent, almost no conversion of the same homogeneous ferricyanide solution with pLys 196 could be observed in neutral conditions ( $\mathrm{pH} 7.4$, Fig. 2c), while complete conversion into 197 ferrocyanide droplets was observed within 15 minutes under acidic conditions ( $\mathrm{pH}$ 4). In both 198 cases, with GSH and $\mathrm{NADH}$, a stoichiometric amount of $\mathrm{S}_{2} \mathrm{O}_{8}{ }^{2-}$ completely dissolved a dispersion 199 of $\mathrm{Fe}(\mathrm{CN})_{6}{ }^{4-}$ droplets, converting $\mathrm{Fe}(\mathrm{CN})_{6}^{4-}$ back into $\mathrm{Fe}(\mathrm{CN})_{6}{ }^{3-}$ within ten minutes. Fig. 2e 200 illustrates the remarkable reversibility of this process: droplets could be generated and dissolved 201 up to eight times, and we were able to carry out identical transitions when starting from either $202 \mathrm{Fe}(\mathrm{CN})_{6}^{4-}$ or $\mathrm{Fe}(\mathrm{CN})_{6}^{3-}$. After eight cycles, the system loses its ability to condense into droplets, 203 which is mainly caused by accumulation of the waste products from GSH or NADH and $\mathrm{S}_{2} \mathrm{O}_{8}{ }^{2-}$.

204 The level of redox control over droplet generation shown in Figure 2 has not been achieved before 205 and holds great promise for the development of dynamic protocell models.

206 For use of these ferricyanide coacervates as potential oxidizing hubs in which oxidation 207 reactions could be localized, it is important to know where the redox reactions utilized in Fig. 2 208 take place. We can take advantage of the fluorescence of common redox-active metabolites such 209 as NADPH, in combination with the observed $\mathrm{pH}$ dependence, to monitor the conversion ferri- and 210 ferrocyanide. We incubated ferricyanide/(Arg) 10 coacervates (8 mM ferricyanide/24 mM Arg) with $2112 \mathrm{mM}$ NADPH at neutral $\mathrm{pH}$ and observed clear NADPH fluorescence inside the coacervates, 212 indicating that NADPH is sequestered by the coacervates (Supplementary Fig. 7). At this $\mathrm{pH}$, the 213 reduction of ferricyanide by NADPH is suppressed (Fig. 2c), which allows for equilibration and 214 focusing of the microscope. We then decreased the outer $\mathrm{pH}$ by addition of a fixed amount of acid 
215 and monitored how the redox reaction progressed and led to the rapid disappearance of the

216 fluorescence of NADPH within 2 min. (Supplementary Fig. 7). Interestingly, the fluorescence

217 intensity first disappeared from the center of the coacervate droplets (Supplementary Fig. 7),

218 indicating that the oxidation of NADPH to non-fluorescence $\mathrm{NADP}^{+}$by ferricyanide took place 219 predominantly inside the droplets instead of in solution, ${ }^{18}$ in which case an exchange of

$220 \mathrm{NADPH} / \mathrm{NADP}^{+}$and reduction of fluorescence at the droplet interface would be expected. Taken

221 together, these data show our ferricyanide-based coacervates are redox-active compartments that

222 can locally oxidize sequestered metabolites.

\section{Fiber self-assembly inside ferricyanide-based coacervates}

225 We further investigated if the redox activity of ferricyanide-based coacervates could lead to spatially controlled higher order assembly. Self-assembly of filaments inside or at the periphery of (proto)cellular compartments is key to many transport processes, locomotion and division. ${ }^{39,40}$

228 Spatially controlled assembly of analogous model filaments in cell-like compartments is therefore

229 an interesting goal in protocell and synthetic cell research. ${ }^{40-42}$ We selected an amino acid 230 derivative benzoyl cysteine as precursor for filaments (Fig. 3). $N, N^{\prime}$-dibenzoyl-L-cystine (DBC) is 231 a well-known redox-active supramolecular gelator, ${ }^{43,44}$ which has been used to make filaments in

232 aqueous solution at low $\mathrm{pH}$. To create the precursor form, we reduced the water soluble $\mathrm{DBC}^{2-}$ at $233 \mathrm{pH} 7$ with 1 equivalent of dithiothreitol (DTT) to give non-active monomer $N$-benzoyl-L-cysteine

$234\left(\mathrm{BC}^{-}\right)$, which is highly soluble at both high and low $\mathrm{pH}$ and does not stack to form fibers.

235 We flushed a solution of $\mathrm{BC}^{-}$(with dye Nile red) in a microchamber containing 236 ferricyanide/(Arg) ${ }_{10}$ coacervates (final concentration: $10 \mathrm{mM} \mathrm{BC}^{-}, 4 \mathrm{mM}$ ferricyanide, $12 \mathrm{mM}$ 237 (Arg) $)_{10}$ (monomer basis)) at $\mathrm{pH}$ 7. Upon addition of the coacervates, we observed a gradual increase 238 in the total fluorescent intensity inside coacervates, as newly formed non-active monomer 2- 
239 charged $\mathrm{DBC}^{2-}$, which weakly binds to Nile red, partitioned in the coacervates (Fig. 3a). At this

$240 \mathrm{pH}$, no fibers are formed, and the non-active $\mathrm{DBC}^{2-}$ monomers are distributed homogeneously

241 inside the coacervates. We note that ferricyanide/peptide coacervates without $\mathrm{DBC}^{2-}$ do not

242 sequester Nile red (Supplementary Fig. 4h). Upon addition of BC to an aqueous dispersion of

243 ferricyanide/( $\operatorname{Arg})_{10}$ coacervates at $\mathrm{pH} 3$ (below the $\mathrm{p} K_{\mathrm{a}}$ of the carboxylate groups of $\mathrm{DBC}, \mathrm{p} K_{\mathrm{a}} \sim$

$2443.6),{ }^{44}$ we observed oxidized, bright fluorescent DBC filaments assembled into shells around the

245 coacervate droplets, and several bundles of filaments present inside the droplets (Fig. 3b). The

246 bundled filaments inside the coacervates are clearly visible in transmission, and are strongly stained

247 by Nile red. These bundles can be seen to pierce the interface of the coacervate droplets. In control

248 with ferricyanide but without polycations, we observed a fiber dispersion without clear bundling

249 or formation of shells (Fig. 3c).

250

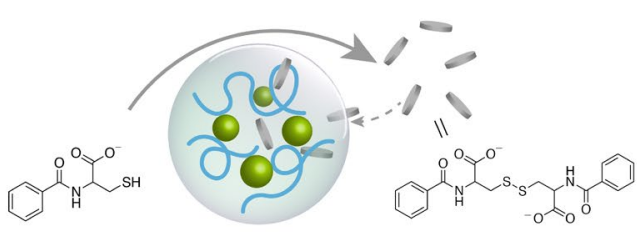

BC- $\mathrm{pH}^{7}$

Non-active monomer, DBC
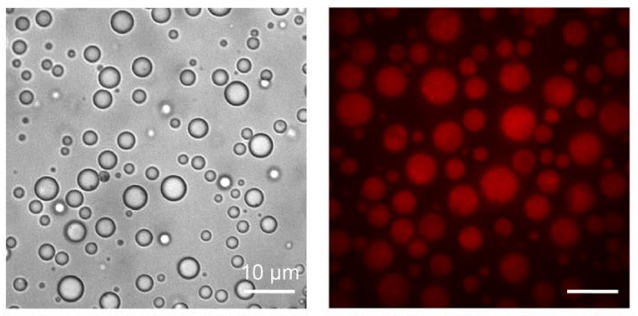

b
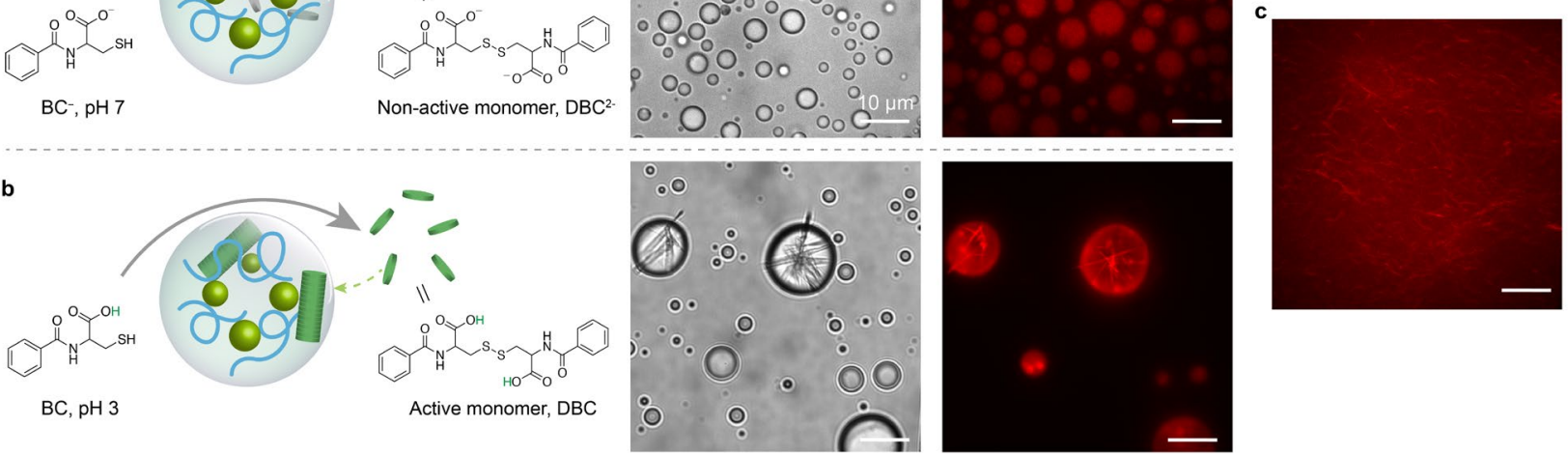

Figure 3. (a) Confocal micrographs of ferricyanide/(Arg) ${ }_{10}$ protocells (with dye, Nile red), time series of representative addition of BC. (c) Confocal micrographs of fiber formation after adding ferricyanide into BC solution. 
A similar type of interfacial filament assembly has been observed for actin filaments and

256 peptide-based pLys/pGlu coacervates before. ${ }^{41}$ We also observed that different peptides lead to

257 altered fiber assembly: in the case of ferricyanide/(Lys(Me $\left.)_{3}\right)_{20}$ and ferricyanide/(Lys(Me $\left.)_{3}\right)_{30}$

258 coacervates, the fibers preferentially localized inside and at the interface of the coacervates

259 (Supplementary Fig. 8a-b). Interestingly, in the case of ferricyanide/(Lys) $)_{30}$ coacervates, the fibers

260 did not remain confined inside the coacervates, but grew out into the surrounding environment,

261 giving an aster-like shape (Supplementary Fig. 8c). In short, these findings show that ferricyanide

262 coacervates can drive the formation of self-assembled filaments via oxidation of the filament

263 precursors.

265 Amide bond formation through amino thioacid oxidation in coacervate protocells

266 Having established the potential of ferricyanide-based coacervates as oxidizing hubs for redox-

267 active guest molecules, we sought to use the oxidizing potential to synthesize peptides by 268 catalyzing the formation of amide bonds. Ferricyanide has been described as a prebiotically

269 abundant oxidizing agent, ${ }^{33}$ and has been used to activate amino thioacids by oxidation to facilitate

270 the formation of an amide bond upon reaction with nucleophilic aminonitriles and amino acids. ${ }^{6-}$

2719,34 However, the oxidative aminoacylation of thioacids is usually performed with high reactant and

272 ferricyanide concentrations (sometimes close to $100 \mathrm{mM}$ ), which could have been difficult to reach

273 everywhere on Early Earth. Therefore, the highly concentrated ferricyanide coacervate droplets

274 could be interesting model compartments for prebiotic amide bond formation (Fig. 4a). As a proof

275 of principle, we first prepared ferricyanide coacervate droplets by direct mixing of aqueous

276 solutions of ferricyanide and trimethylated poly-L-lysine (pLys $\left.(\mathrm{Me})_{3}\right)$ at charge stoichiometry. The

277 pLys $(\mathrm{Me})_{3}$ was chosen to avoid the $\varepsilon$-coupling of Lys- $\mathrm{NH}_{2}$. To study the amide bond formation in 278 coacervate droplets, we incubated $N$-acetyl-glycine thioacid (Ac-Gly-SH) (8 mM) with Gly (3 eq.) 
279 in ferricyanide (1 eq.)/ $\mathrm{pLys}(\mathrm{Me})_{3}$ coacervates dispersion at $\mathrm{pH}$ 9. The consumption of Ac-Gly-SH 280 and formation of the ligation product Ac-Gly-Gly-OH was monitored with ${ }^{1} \mathrm{H}$ NMR (Fig. 4b).

281 Interestingly, we found a yield of Ac-Gly-Gly-OH of up to $80 \%$ after 3 days in the presence 282 of coacervates, while in dilute phase at most $5 \%$ of ligation product was observed. In controls with 283 peptides but without ferricyanide, we found no ligation product (Supplementary Fig. 9), and also 284 in the presence of ferrocyanide/pLys coacervate droplets, no ligation product was observed 285 (Supplementary Fig. 10). The significantly higher yield in the presence of coacervates can be 286 attributed to the high local ferricyanide concentration inside coacervate droplets and implies that 287 the ligation reaction takes place predominantly inside the coacervates. Ferricyanide-based 288 coacervate droplets can thus act as microreactors that enhance the rates of oxidative aminoacylation.

289 We found that the ligation reaction was most enhanced in droplets formed from shorter peptides, $290\left(\operatorname{Lys}(\mathrm{Me})_{3}\right)_{20}$ and $\left(\operatorname{Lys}(\mathrm{Me})_{3}\right)_{30}$, compared to longer pLys(Me) ${ }_{3}$ (Fig. 4c). We attribute this to the 291 lower multivalency of the polycation, which results in weaker complexation with the ferricyanide, 292 making it more available for the reaction with the amino thioacids.

293 From an origins of life perspective, it is interesting to explore the selectivity of aminoacylation 294 when more than one amino acid can react with an available amino thioacid inside coacervate 295 droplets. Instead of only glycine (Gly), stoichiometric (1:1) competition reactions between glycine 296 and glutamic acid (Glu), Alanine (Ala), and Phenylalanine (Phe) were investigated. All competition 297 reactions demonstrated a significant selectivity for one of the ligation products at $\mathrm{pH} 9$ 298 (Supplementary Table 1). For example, when we added both glycine (12 mM) and glutamic acid 299 (12 mM) to ferricyanide-based droplets (8 mM ferricyanide) containing Ac-Gly-SH (8 mM), we 300 observed a strong selectivity for peptide ligation with glycine. Glutamic acid alone yields the Ac301 Gly-Glu-OH dipeptide in similar yields as glycine (Fig. 4d, Supplementary Fig. 11a), but when 
a
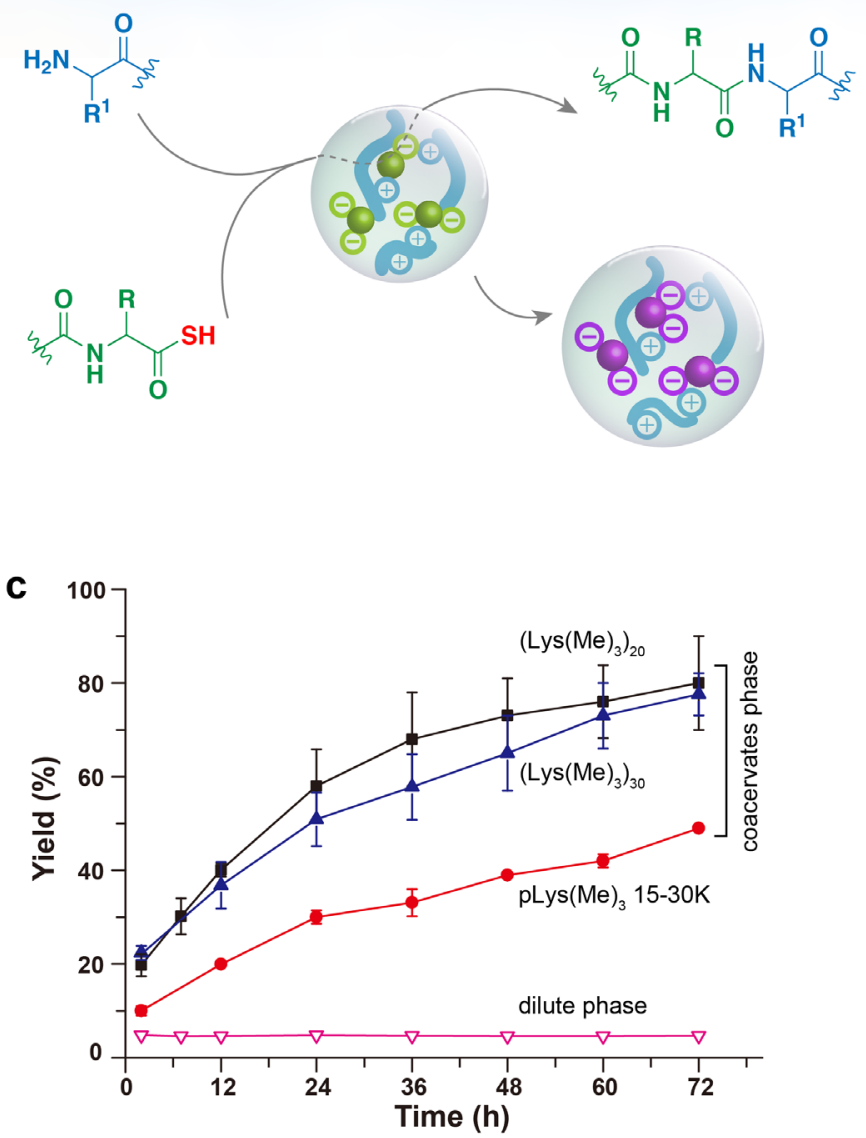
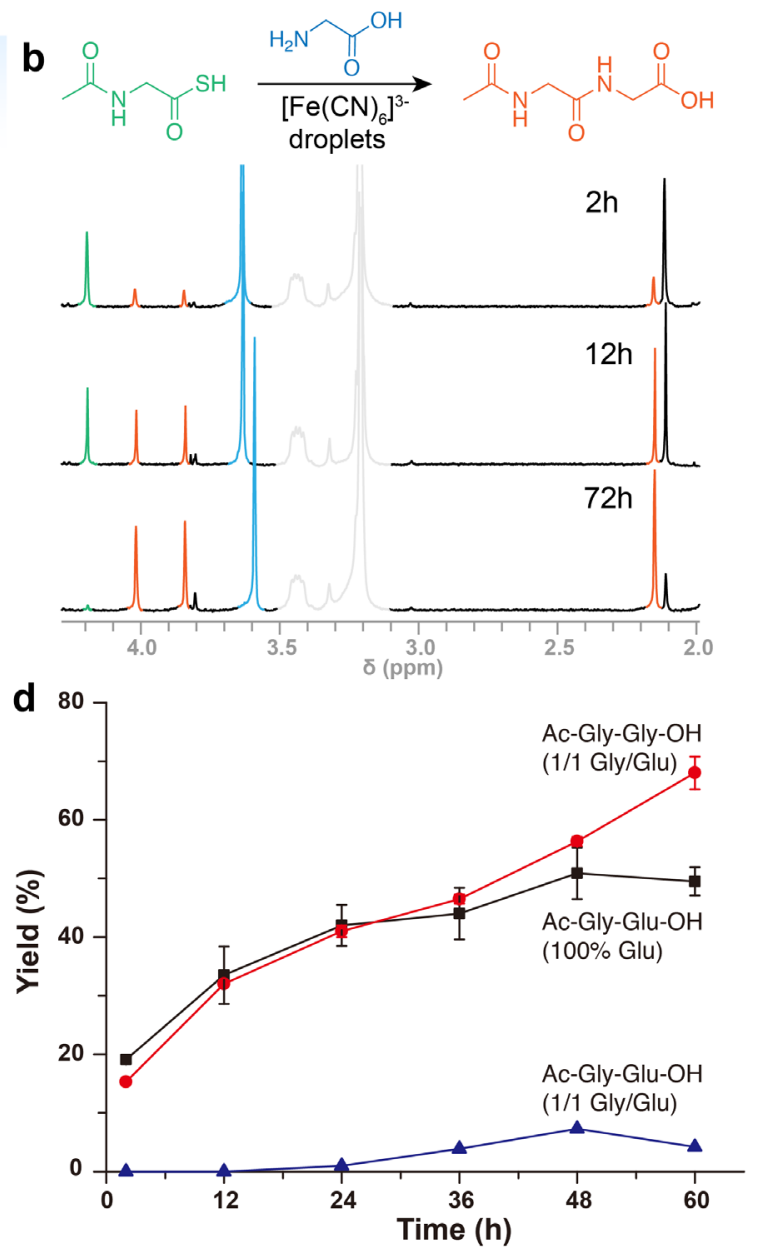

Figure 4. Peptide bond formation in ferricyanide-based droplets. (a) Schematic illustration of ferricyanide-based droplets as microreactors for peptide ligation in water. (b) ${ }^{1} \mathrm{H}$ NMR spectrum showing the peptide ligation reaction of $\mathrm{N}$-acetyl-glycine thioacid Ac-Gly-SH (8mM, green) and Gly-OH (3 equiv., blue) with ferricyanide/plys(Me) 3 (gray) coacervates (1 equiv., $\mathrm{pH} 9$, room temperature) to yield Ac-Gly-Gly-OH (orange). (c) Plot of \% ligation products vs time for peptide ligation reaction in dilute phase or ferricyanide-based coacervate droplets. (d) Plot of selective peptide bond formation within coacervate droplets.

310 glycine and glutamic acid were incubated together in a 1:1 ratio with Ac-Gly-SH in

311 (Lys(Me) $\left.)_{3}\right)_{20} /$ ferricyanide coacervates, more than $90 \%$ of the ligation products was Ac-Gly-Gly-

312 OH (Fig. 4d, Supplementary Fig. 11b). Glycine thus outcompeted glutamic acid very effectively

313 in the ligation process. We reasoned that the activity of glycine inside coacervates is higher than

314 glutamic acid. Although glycine and glutamic acid have similar nucleophilicities ${ }^{45}$ glutamic acid 
315 is bound more strongly to the cationic lysine residues inside the coacervate and therefore has a

316 lower activity. As a result, glycine reacts faster than glutamic acid inside the coacervate

317 environment and the outcome we observe is an example of kinetic pathway selection, caused by

318 the local protocell environment. Likewise, we observed selective incorporation of glycine in 319 mixtures with alanine ( $83 \%$ Gly $/ 17 \%$ Ala) and phenylalanine (78\% Gly/22\% Phe) (Supplementary

320 Table 1), because the latter have a slightly stronger interaction with the peptide backbone in the

321 coacervates. Alanine is incorporated with slight preference over glutamic acid ( $60 \% \mathrm{Ala} / 40 \% \mathrm{Glu})$,

322 but surprisingly, glutamic acid is incorporated more very slightly effectively than phenylalanine $323(63 \% \mathrm{Glu} / 37 \% \mathrm{Phe})$.

324 The products of the above reactions with simple amino (thio)acids are small molecules that 325 interact only weakly with the coacervates. Therefore, they can quickly escape the protocell and the 326 evolutionary advantage for the protocells is limited. To establish a feedback between the formation 327 of amide bonds inside protocells and protocell fitness, we sought to retain the reaction product 328 inside the coacervates. We tested if the scaffold peptides used to form the coacervates could 329 themselves react as nucleophiles with oxidized aminothioacids. We added Ac-Phe-SH or Phe-SH $330(4 \mathrm{mM})$ to ferricyanide $(4 \mathrm{mM}) /$ pLys $(12 \mathrm{mM})$ droplets, leading to $\varepsilon-\mathrm{NH}_{2}$ ligation of the protocell 331 building blocks (pLys) (Fig. 5a, Supplementary Fig. 12). Anchoring of the aromatic phenylalanine 332 to the pLys scaffold makes the coacervate interior more hydrophobic, and leads to additional 333 cation- $\pi$ interactions between the coacervate components, both of which result in an expected 334 increase in salt resistance. ${ }^{27}$ Indeed, we found that ferricyanide/pLys-g-Phe coacervates remain 335 stable far beyond the CSC of the original ferricyanide/pLys coacervates. Fig. $5 \mathrm{~b}$ shows the turbidity 336 of the coacervates for increasing the salt concentrations. In the control sample, to which we added 337 GSH (4 mM) instead of an amino thioacid to reduce the ferricyanide, the turbidity decreases and 
338 reaches background levels around $180 \mathrm{mM} \mathrm{NaCl}$, where all droplets had disappeared completely

339 (Fig. 5c). In contrast, when Ac-Phe-SH/Phe-SH was added and reacted with the $\varepsilon-\mathrm{NH}_{2}$ of pLys,

340 the turbidity transition shifted to a significantly higher salt concentration and the absolute intensity

341 of the plateau at high salt concentration was higher (Fig. 5b). Even at high salt concentrations (220

$342 \mathrm{mM}$ ), above the original CSC, microscope images show the presence of gel-like droplets, which

343 do not fuse (Fig. 5c). These condensates have an increased salt resistance and a more gel-like

344 consistency as a result of the attachment of an aromatic residue to the pLys side chains.

a
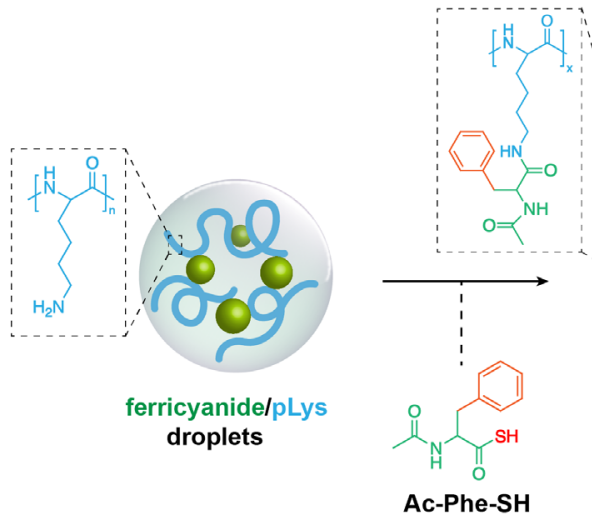

C

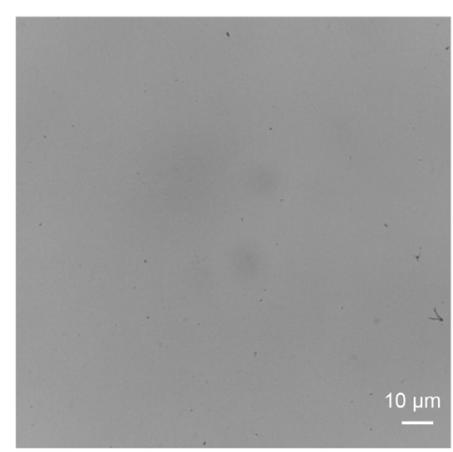

$\mathrm{GSH}, \mathrm{C}_{\mathrm{NaCl}}(220 \mathrm{mM})$
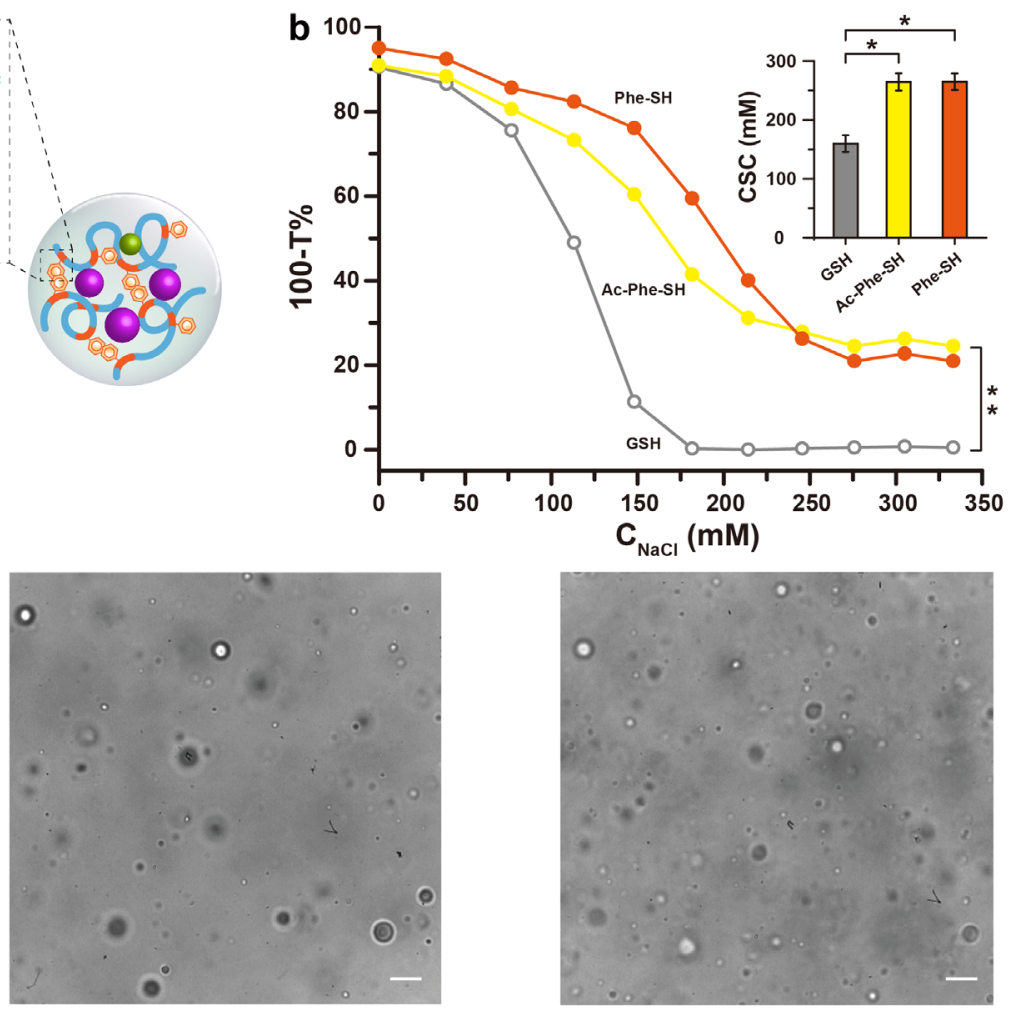

Ac-Phe-SH, $\mathrm{C}_{\mathrm{NaCl}}(220 \mathrm{mM})$

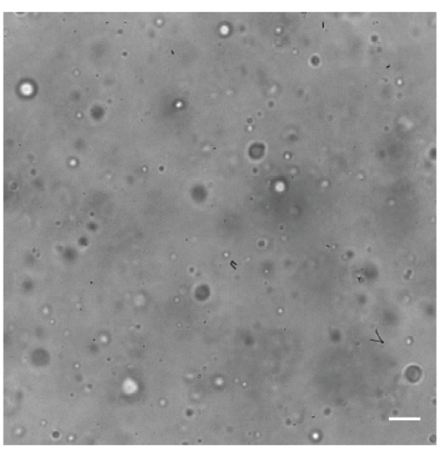

Phe-SH, $\mathrm{C}_{\mathrm{NaCl}}(220 \mathrm{mM})$

Figure 5. (a) Schematic representation of pLys $\varepsilon-\mathrm{NH}_{2}$ ligation in coacervates. (b) Salt resistance of coacervates with Ac-Phe-SH/Phe-SH ligation with droplets building blocks (pLys). Insets show the significantly differents of the corresponding CSC, or the turbidity plateau. (c) Optical microscope images illustrating samples corresponding to the high salt concentration $(220 \mathrm{mM})$ points in (b). 


\section{Conclusion}

352 In summary, we developed a protocell model based on prebiotically relevant ferricyanide as redox-

353 active species. Membrane-free droplet compartments were spontaneous assembled in the presence

354 of short cationic peptides and the assembly of ferri/ferrocyanide-peptide droplets can be regulated

355 by redox chemistry and salt concentration. Ferricyanide-peptide droplets can act as oxidizing hubs

356 for metabolites, such as $\mathrm{NAD}(\mathrm{P}) \mathrm{H}$ and GSH, filament stacking element like benzoyl cysteine, and

357 amino thioacids as potential prebiotic precursors of amino acids. We demonstrate that the oxidation

358 of amino thioacids by ferricyanide coacervates can be used to drive aminoacylation, resulting in

359 the formation of new peptide bonds. The amino acid ligation is enhanced in coacervate dispersions

360 compared to the surrounding dilute phase due to the local high ferricyanide concentration. The

361 coacervate environment imposes a selection pressure that results in kinetic pathway selection and

362 a strong, preferential incorporation of certain amino acids. Finally, this strategy can be used to

363 create self-reinforcing coacervates, in which hydrophobic amino acid residues are ligated to the

364 coacervate building blocks and enhance their stability. Our results show that prebiotically relevant

365 ferricyanide-based coacervate protocells are versatile oxidizing hubs that exist in aqueous solution,

366 in which metabolites can be sequestered and peptides synthetized. These results provide an

367 important step towards prebiotically plausible integration of chemical processes in cellular 368 compartments.

370 Methods

371 A full description of materials and methods used in this work is given in the Supplementary 372 Information. 


\section{Acknowledgements}

375 This work was financially supported by the European Research Council (ERC) under grant number

376 851963. The authors would like to thank Karina Nakashima for synthesis of the $N$-acetyl glycine

377 thioacid and helpful discussions about the project goals, Haibin Qian for preliminary experiments

378 on EDC-mediated amide bond formation in pGlu/pLys(Me $)_{3}$ coacervates and the methylation

379 protocol for pLys, and Tiemei Lu for help with preparing the methylated pLys.

380

$381 \quad$ Additional information

382 Supplementary information accompanies this paper.

383

384 Author information

385 Corresponding authors

386 *Email: e.spruijt@science.ru.nl

387 *Email: junyouwang@ecust.edu.cn

388

389 Competing interests

390 The authors declare no competing financial interests.

391

392

\section{References}

1. Nissen, P., Hansen, J., Ban, N., Moore, P. B. \& Steitz, T. A. The Structural Basis of Ribosome Activity in Peptide Bond Synthesis. Science 289, 920-930 (2000).

2. Goswami, A. \& Lanen, S. G. V. Enzymatic strategies and biocatalysts for amide bond formation: tricks of the trade outside of the ribosome. Mol. Biosyst. 11, 338-353 (2015).

397 3. Joyce, G. F. \& Szostak, J. W. Protocells and RNA Self-Replication. Cold Spring Harb. Perspect. Biol. 10, a034801 (2018).

4. Li, M., Huang, X., Tang, T.-Y. D. \& Mann, S. Synthetic cellularity based on non-lipid micro-compartments and protocell models. Curr. Opin. Chem. Biol. 22, 1-11 (2014). 
5. Abbas, M., Lipiński, W. P., Wang, J. \& Spruijt, E. Peptide-based coacervates as biomimetic protocells. Chem. Soc. Rev. 50, 3690-3705 (2021).

6. Maurel, M.-C. \& Orgel, L. E. Oligomerization of $\alpha$-Thioglutamic Acid. Orig. Life Evol. Biosph. 30, $423-430$ (2000).

7. Canavelli, P., Islam, S. \& Powner, M. W. Peptide ligation by chemoselective aminonitrile coupling in water. Nature 571, 546-549 (2019).

8. Okamoto, R. et al. Regioselective $\alpha$-Peptide Bond Formation Through the Oxidation of Amino Thioacids. Biochemistry 58, 1672-1678 (2019).

9. Liu, R. \& Orgel, L. E. Oxidative acylation using thioacids. Nature 389, 52-54 (1997).

10. McLean, E. B. \& Lee, A.-L. Golden potential. Nat. Chem. 11, 760-761 (2019).

11. Sokolova, E. et al. Enhanced transcription rates in membrane-free protocells formed by coacervation of cell lysate. Proc. Natl. Acad. Sci. (2013) doi:10.1073/pnas.1222321110.

12. Drobot, B. et al. Compartmentalised RNA catalysis in membrane-free coacervate protocells. Nat. Commun. 9 , 3643 (2018).

13. Poudyal, R. R. et al. Template-directed RNA polymerization and enhanced ribozyme catalysis inside membraneless compartments formed by coacervates. Nat. Commun. 10, 490 (2019).

14. Mason, A. F. et al. Mimicking Cellular Compartmentalization in a Hierarchical Protocell through Spontaneous Spatial Organization. ACS Cent. Sci. 5, 1360-1365 (2019).

15. Altenburg, W. J. et al. Programmed spatial organization of biomacromolecules into discrete, coacervate-based protocells. Nat. Commun. 11, 6282 (2020).

16. Abbas, M., Lipiński, W. P., Nakashima, K. K., Huck, W. T. S. \& Spruijt, E. Short peptide synthon for liquidliquid phase separation. Nat. Chem. doi:10.1038/s41557-021-00788-x.

17. Koga, S., Williams, D. S., Perriman, A. W. \& Mann, S. Peptide-nucleotide microdroplets as a step towards a membrane-free protocell model. Nat. Chem. 3, 720-724 (2011).

18. Donau, C. et al. Active coacervate droplets as a model for membraneless organelles and protocells. Nat. Commun. 11, 5167 (2020).

19. Cakmak, F. P., Choi, S., Meyer, M. O., Bevilacqua, P. C. \& Keating, C. D. Prebiotically-relevant low polyion multivalency can improve functionality of membraneless compartments. Nat. Commun. 11, 5949 (2020).

20. Lu, T. \& Spruijt, E. Multiphase Complex Coacervate Droplets. J. Am. Chem. Soc. 142, 2905-2914 (2020).

21. Mountain, G. A. \& Keating, C. D. Formation of Multiphase Complex Coacervates and Partitioning of Biomolecules within them. Biomacromolecules 21, 630-640 (2020).

22. Zhang, Y. et al. Giant Coacervate Vesicles As an Integrated Approach to Cytomimetic Modeling. J. Am. Chem. Soc. 143, 2866-2874 (2021).

23. Nakashima, K. K., van Haren, M. H. I., André, A. A. M., Robu, I. \& Spruijt, E. Active coacervate droplets are protocells that grow and resist Ostwald ripening. Nat. Commun. 12, 3819 (2021).

24. Love, C. et al. Reversible pH-Responsive Coacervate Formation in Lipid Vesicles Activates Dormant Enzymatic Reactions. Angew. Chem. Int. Ed. 59, 5950-5957 (2020).

25. Last, M. G. F., Deshpande, S. \& Dekker, C. pH-Controlled Coacervate-Membrane Interactions within Liposomes. ACS Nano 14, 4487-4498 (2020).

26. Deng, N.-N. \& Huck, W. T. S. Microfluidic Formation of Monodisperse Coacervate Organelles in Liposomes. Angew. Chem. Int. Ed. 56, 9736-9740 (2017).

27. Lu, T., Nakashima, K. K. \& Spruijt, E. Temperature-Responsive Peptide-Nucleotide Coacervates. J. Phys. Chem. B 125, 3080-3091 (2021).

28. Martin, N. et al. Photoswitchable Phase Separation and Oligonucleotide Trafficking in DNA Coacervate Microdroplets. Angew. Chem. Int. Ed. 58, 14594-14598 (2019).

29. Aumiller, W. M. \& Keating, C. D. Phosphorylation-mediated RNA/peptide complex coacervation as a model for intracellular liquid organelles. Nat. Chem. 8, 129-137 (2016).

30. Nakashima, K. K., Baaij, J. F. \& Spruijt, E. Reversible generation of coacervate droplets in an enzymatic network. Soft Matter 14, 361-367 (2018).

31. Deshpande, S. et al. Spatiotemporal control of coacervate formation within liposomes. Nat. Commun. 10, 1800 (2019).

32. Bungenberg de Jong, H. G. \& Kruyt, H. R. Coacervation (Partial miscibility in colloid systems). Proc Kon Ned Akad Wet 32, 849-856 (1929).

33. Keefe, A. D. \& Miller, S. L. Was ferrocyanide a prebiotic reagent? Orig. Life Evol. Biosph. 26, 111-129 (1996).

34. Leman, L., Orgel, L. \& Ghadiri, M. R. Carbonyl Sulfide-Mediated Prebiotic Formation of Peptides. Science 306, 283-286 (2004). 
35. Coggins, A. J. \& Powner, M. W. Prebiotic synthesis of phosphoenol pyruvate by $\alpha$-phosphorylation-controlled triose glycolysis. Nat. Chem. 9, 310-317 (2017).

36. Sokalingam, S., Raghunathan, G., Soundrarajan, N. \& Lee, S.-G. A Study on the Effect of Surface Lysine to Arginine Mutagenesis on Protein Stability and Structure Using Green Fluorescent Protein. PLOS ONE 7 , e40410 (2012).

37. Choi, E.-Y. et al. Electrochemical Characteristics of Polyelectrolyte Brushes with Electroactive Counterions. Langmuir 23, 10389-10394 (2007).

38. Wang, J., Cohen Stuart, M. A. \& van der Gucht, J. Phase Diagram of Coacervate Complexes Containing Reversible Coordination Structures. Macromolecules 45, 8903-8909 (2012).

39. Miyazaki, M., Chiba, M., Eguchi, H., Ohki, T. \& Ishiwata, S. Cell-sized spherical confinement induces the spontaneous formation of contractile actomyosin rings in vitro. Nat. Cell Biol. 17, 480-489 (2015).

40. te Brinke, E. et al. Dissipative adaptation in driven self-assembly leading to self-dividing fibrils. Nat. Nanotechnol. 13, 849-855 (2018).

41. McCall, P. M. et al. Partitioning and Enhanced Self-Assembly of Actin in Polypeptide Coacervates. Biophys. J. 114, 1636-1645 (2018).

42. Fanalista, F., Deshpande, S., Lau, A., Pawlik, G. \& Dekker, C. FtsZ-Induced Shape Transformation of Coacervates. Adv. Biosyst. 2, 1800136 (2018).

43. Boekhoven, J., Hendriksen, W. E., Koper, G. J. M., Eelkema, R. \& Esch, J. H. van. Transient assembly of active materials fueled by a chemical reaction. Science 349, 1075-1079 (2015).

44. Wojciechowski, J. P., Martin, A. D. \& Thordarson, P. Kinetically Controlled Lifetimes in Redox-Responsive Transient Supramolecular Hydrogels. J. Am. Chem. Soc. 140, 2869-2874 (2018).

45. Brotzel, F. \& Mayr, H. Nucleophilicities of amino acids and peptides. Org. Biomol. Chem. 5, 3814-3820 (2007). 


\title{
Supplementary information
}

\section{Selective amide bond formation in redox-active coacervate protocells}

\author{
Jiahua Wang, ${ }^{[a]}$ Manzar Abbas, ${ }^{[a]}$ Junyou Wang ${ }^{*}[\mathrm{~b}]$ and Evan Spruijt ${ }^{*}[\mathrm{a}]$ \\ ${ }^{[a]}$ Radboud University, Institute for Molecules and Materials, Heyendaalseweg 135, 6525 AJ Nijmegen, the Netherlands \\ [b] State Key Laboratory of Chemical Engineering and Shanghai Key Laboratory of Multiphase Materials Chemical \\ Engineering, East China University of Science and Technology, Shanghai 200237, China
}

*Email: e.spruijt@science.ru.nl, or: junyouwang@ecust.edu.cn

\section{Materials and methods}

\section{Materials}

Poly-L-lysine hydrobromide (pLys, 15-30 kDa), potassium ferricyanide, potassium ferrocyanide, glutathione (GSH), DL-dithiothreitol (DTT), tris(hydroxymethyl)aminomethane (Tris), pyranine, and sodium chloride were purchased from Sigma Aldrich and used without further purification. Short polycations (Lys) 10 , (Lys) ${ }_{20}$, (Lys) 30 , (Arg) 10 were purchased form Alamanda Polymers. Nicotinamide adenine dinucleotide (NADH) and nicotinamide adenine dinucleotide phosphate (NADPH) were purchased from Roche. The fluorescently labeled oligonucleotides poly-A ${ }_{15}(\mathrm{Cy} 5-$ $\mathrm{A}_{15}$ ), Poly-rU15 (rU15-Cy3Sp) were purchased from Integrated DNA Technologies (IDT). 


\section{Coacervate formation}

Samples for turbidity measurements were prepared directly into 96-well plates, by adding, respectively, Milli-Q water, Tris buffer ( $\mathrm{pH} 7.4,50 \mathrm{mM}), \mathrm{pLys}$, and ferricyanide or ferrocyanide to a total volume of $100 \mu \mathrm{L}$. Mixing was done by gentle pipetting $(3 \times)$ before each measurement. Samples for the microscopy experiments were prepared in microcentrifuge tubes. After addition of the substrate, a $20 \mu \mathrm{L}$ aliquot was immediately taken for imaging on a glass slide.

\section{Turbidity measurements}

Turbidity measurements were performed in triplicate using a Berthold Tristar (2) LB 942 microplate reader. The temperature was kept constant at $25 \pm 1^{\circ} \mathrm{C}$. The absorbance was measured at $520 \mathrm{~nm}$, where none of the mixture components absorbed significantly. The absorbance of a well filled with the same volume of water was used as a blank. Samples were shaken for $5 \mathrm{~s}$ before every readout. The critical point was determined by extrapolating the first-order derivative at the inflection point to zero turbidity. Note that this critical salt concentration does not take into account ions from other sources than the added $\mathrm{NaCl}$, and the actual critical ionic strength may be slightly higher.

\section{Ferricyanide and ferrocyanide partitioning}

In a typical procedure, the coacervates dispersion was centrifuged in an Eppendorf tube at 3000x g until the dilute phase was transparent under microscope, the coacervate phase had sedimented to the bottom of the cell. The concentration of the ferricyanide/ferrocyanide in the dilute phase was quantitatively analyzed with Uv-vis at wavelength of $320 \mathrm{~nm}$ and $420 \mathrm{~nm}$ (Supplementary Fig. 5).

We measured the volume of the top (dilute) solution and calculated the ferricyanide/ferrocyanide 
concentrations of the top solution from the standard curve, the volume and the moles of ferricyanide /ferrocyanide in coacervate phase can be calculated from the total feed, then the ferricyanide and ferrocyanide concentrations inside the coacervate phase can be obtained.

\section{Confocal fluorescence microscopy}

Optical and fluorescence microscopy images were recorded on an Olympus UIS2 microscope, equipped with a motorized stage (Prior, Optiscan II). Fluorescent images were recorded with an EMCCD camera (Andor, iXon), using illumination from a mercury lamp, an excitation filter of 482/18 nm (Semrock BrightLine) and an emission filter of 525/45 nm (Semrock BrightLine). Samples were loaded into the wells of PLL-g-PEG-functionalized Ibidi $\mu$-slides and closed with a lid (microscopy chambers).

\section{Microscopy chambers preparation}

The Ibidi $\mu$-slides used for imaging were functionalized with PLL-g-PEG to minimize wetting and spreading of the coacervate droplets. Each slide was first activated by oxygen plasma treatment (Diener electronic, Femto). The PLL-g-PEG $(0.01 \mathrm{mg} / \mathrm{mL}$ in $10 \mathrm{mM}$ HEPES buffer, $\mathrm{pH} 7.4)$ solution was added into each well and incubated at room temperature for $1 \mathrm{~h}$. After that, the glass slides were cleaned by rinsing (3 times with 10 mM HEPS, $\mathrm{pH}$ 7.4, 3 times with MQ). The slides were dried by using compressed air.

\section{NMR measurements}

Nuclear Magnetic Resonance (NMR) spectra were measured on a Bruker-AVANCE III $400 \mathrm{MHz}$ spectrometer. 


\section{Mass spectrometry}

Mass spectra were obtained from a Thermo Scientific $^{\mathrm{TM}}$ LCQ Fleet ${ }^{\mathrm{TM}}$ ion trap mass spectrometer with Gemini-NX C18 110A 150 x 2.0 mm column and JEOL Accurate Time of Flight (ToF) instruments, both using linear ion trap electrospray ionisation (ESI).

\section{Synthesis of trimethylated oligolysine and poly-L-lysine}

Trimethylated oligolysines $\left(\operatorname{Lys}(\mathrm{Me})_{3}\right)_{n}$ and poly-L-lysine (pLys(Me $\left.)_{3}\right)$ were prepared from $(\mathrm{Lys})_{\mathrm{n}}$ and pLys, according to literature methods. ${ }^{3}$ Briefly, $2 \mathrm{~mL}$ DMS was added to $50 \mathrm{mg}$ (Lys) , in 20 $\mathrm{mL} \mathrm{H}_{2} \mathrm{O}$ and $6 \mathrm{~mL}$ ethanol. The $\mathrm{pH}$ was adjusted to 9.5 and maintained by the addition of $1 \mathrm{M}$ $\mathrm{NaOH}$. The reaction was considered complete when the $\mathrm{pH}$ remained nearly constant. The pLys(Me) $)_{3}$ was further purified by dialysis against 2 times $1 \mathrm{~L}$ of a $2 \mathrm{M} \mathrm{NaCl}$ aqueous solution and subsequently, 3 times $1 \mathrm{~L}$ of water in a dialysis membrane (MWCO, 3500). After that, the residue was freeze-dried to yield a white powder.

\section{Synthesis of sodium 2-acetamidoethanethioate Ac-Gly-S-Na ${ }^{+}$}

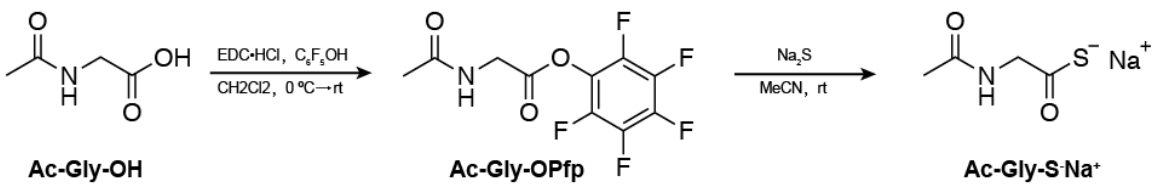

Ac-Gly-S-Na ${ }^{+}$, Ac-Phe-S-Na ${ }^{+}$and Phe-SH were synthesized according to literature methods

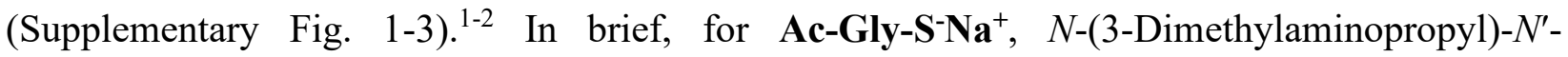
ethylcarbodiimide hydrochloride $(\mathrm{EDC} \cdot \mathrm{HCl})(3.45 \mathrm{~g}, 18.00 \mathrm{mmol})$ was added to a stirring solution of $N$-acetylglycine Ac-Gly-OH (943 mg, $6.00 \mathrm{mmol})$ and pentafluorophenol (1.22 g, $6.60 \mathrm{mmol})$ in $\mathrm{CH}_{2} \mathrm{Cl}_{2}(25 \mathrm{~mL})$ at $0{ }^{\circ} \mathrm{C}$. The reaction mixture was allowed to warm to room temperature and stirred for $16 \mathrm{~h}$. The resultant homogenous solution was diluted with $\mathrm{CH}_{2} \mathrm{Cl}_{2}(20 \mathrm{~mL})$ and washed 
with water $(2 \times 20 \mathrm{~mL}), \mathrm{NaHCO}_{3}$ (sat., 2 × $\left.20 \mathrm{~mL}\right)$ and brine $(20 \mathrm{~mL})$. The organic layer was dried over $\mathrm{Na}_{2} \mathrm{SO}_{4}$, filtered, and concentrated in vacuo to give $N$-acetylglycine pentafluorophenyl ester Ac-Gly-OPfp, which was used immediately without further purification. The crude Ac-Gly-OPfp was resuspended in anhydrous acetonitrile $(15 \mathrm{~mL})$ and stirred vigorously with anhydrous sodium sulfide (1.1 equiv) under argon atmosphere for $6 \mathrm{~h}$ at room temperature. The resultant precipitate was isolated by centrifugation and washed with diethyl ether $(3 \times 10 \mathrm{~mL})$ and lyophilized to yield Ac-Gly-S-Na ${ }^{+}$as a white solid (350 mg). ${ }^{1} \mathrm{H}$ NMR (400 MHz, D $\left.2 \mathrm{O}\right)$ : d 4.08 (s, 2H, (C2)-H $), 1.97$ (s, 3H, $\left(\mathrm{COCH}_{3}\right)$. HRMS-ESI [M-H] $]^{-}$calcd. for $\left[\mathrm{C}_{4} \mathrm{H}_{7} \mathrm{NO}_{2} \mathrm{~S}-\mathrm{H}\right]^{-}:$: 132.0119; observed: 132.0117.

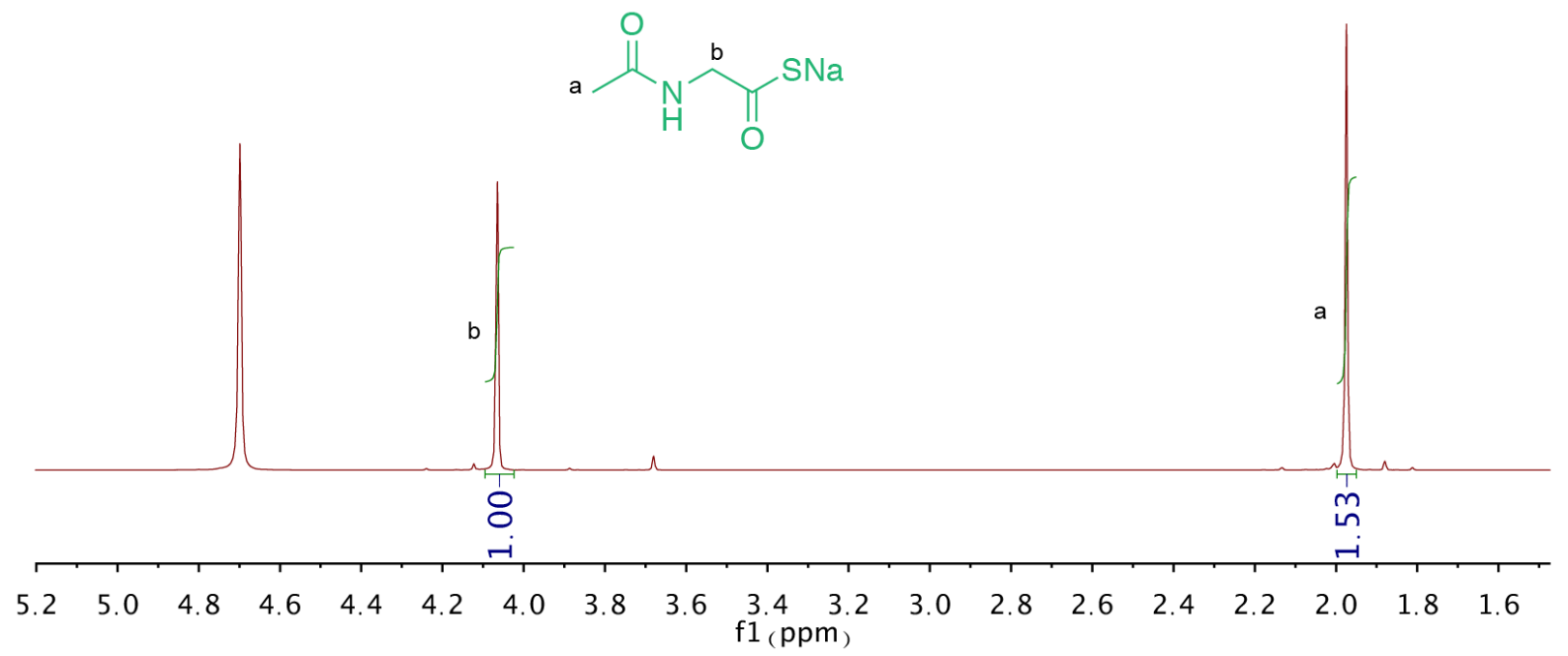

Supplementary Figure 1. ${ }^{1} \mathrm{H}-\mathrm{NMR}$ spectrum of Ac-Gly-S-Na ${ }^{+}\left(400 \mathrm{MHz}, \mathrm{D}_{2} \mathrm{O}\right)$. 


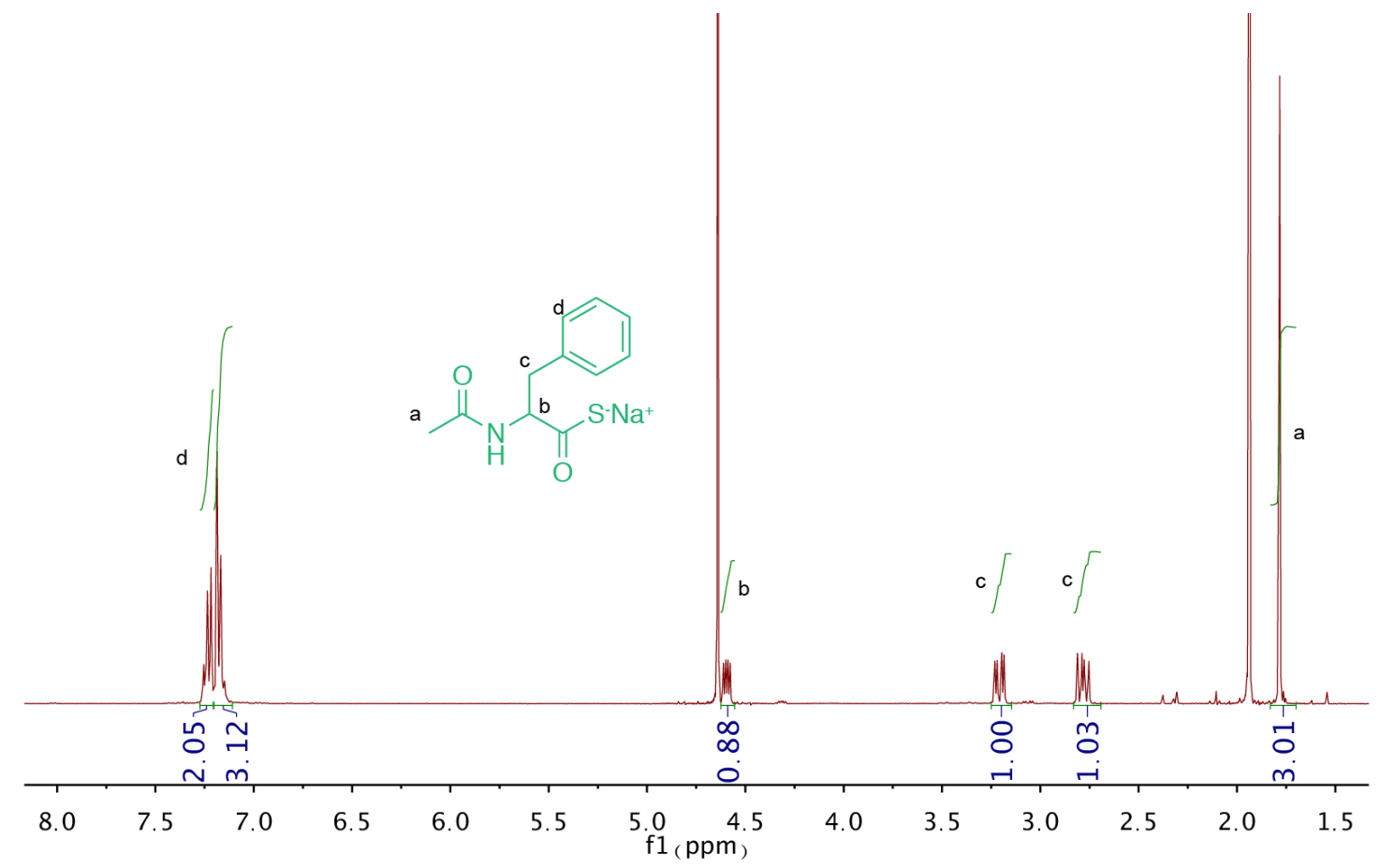

Supplementary Figure 2. ${ }^{1} \mathrm{H}-\mathrm{NMR}$ spectrum of Ac-Phe-S-Na ${ }^{+}\left(400 \mathrm{MHz}, \mathrm{D}_{2} \mathrm{O}\right)$

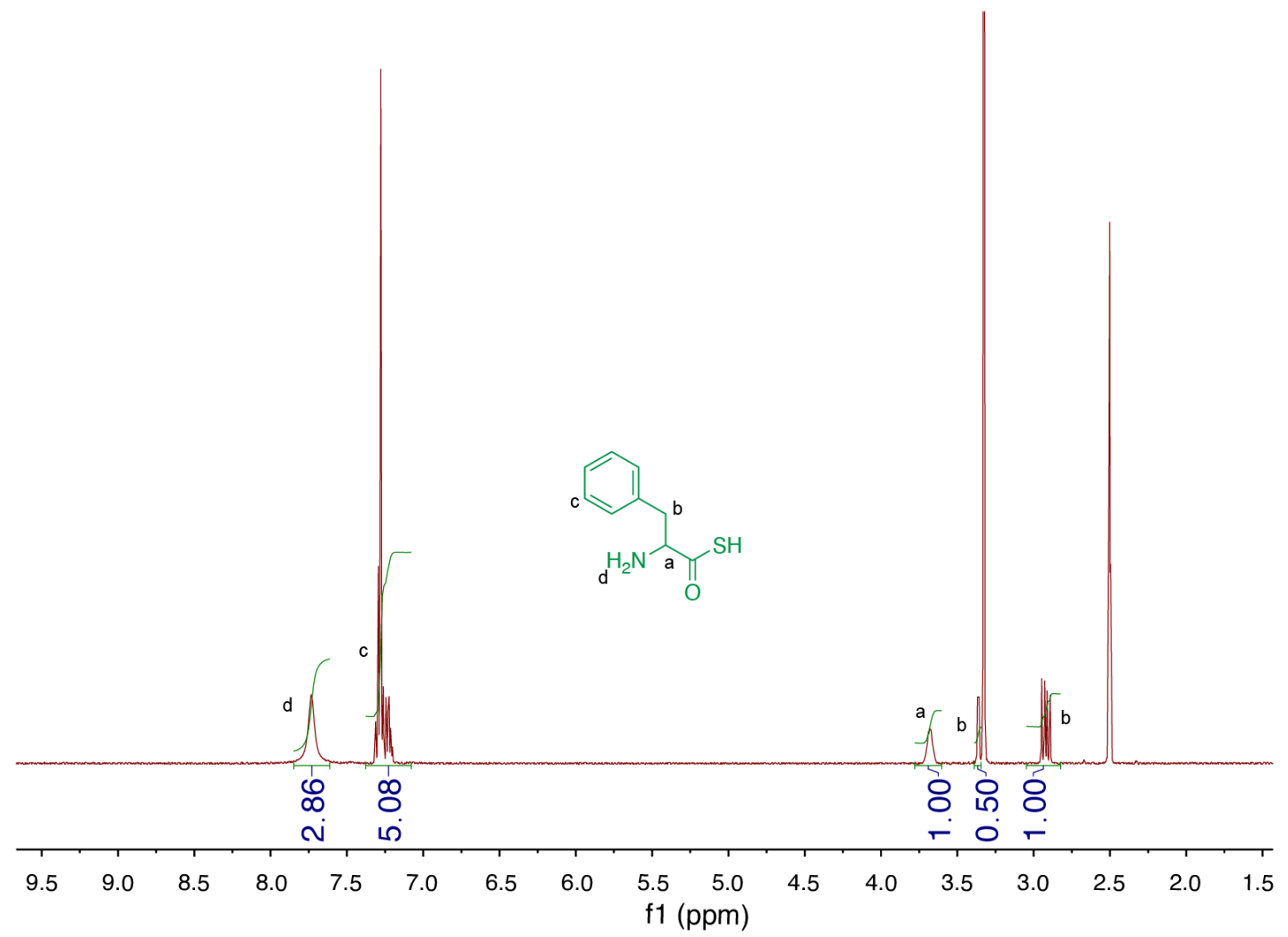

Supplementary Figure 3. ${ }^{1} \mathrm{H}-\mathrm{NMR}$ spectrum of Phe-SH (400 MHz, DMSO-D6). 


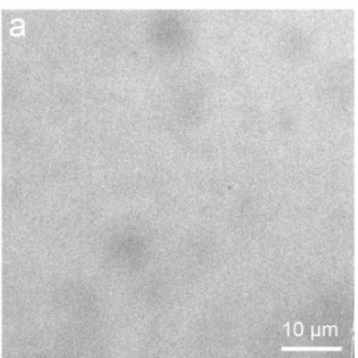

ferricyanide/ (Lys) 20

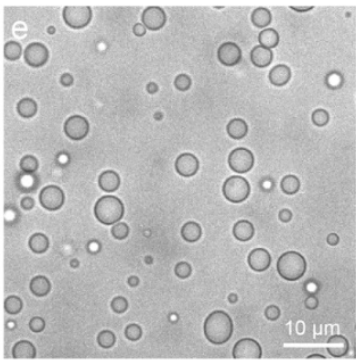

ferricyanide/ (Arg)

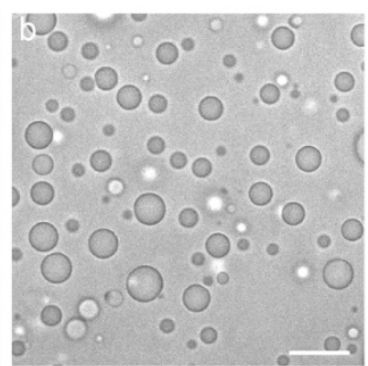

ferricyanide/ (Lys $\left.(\mathrm{Me})_{3}\right)_{20}$

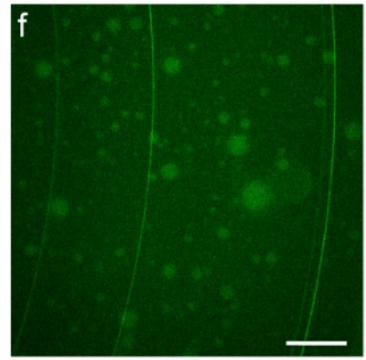

ferricyanide/ (Arg) with dye (NADH)

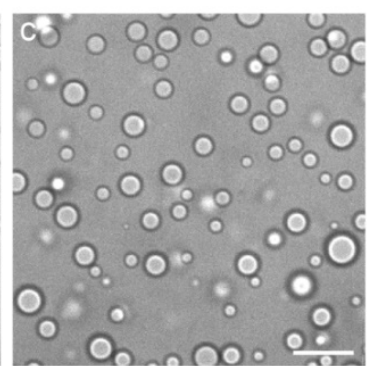

ferricyanide/ (Lys) 30

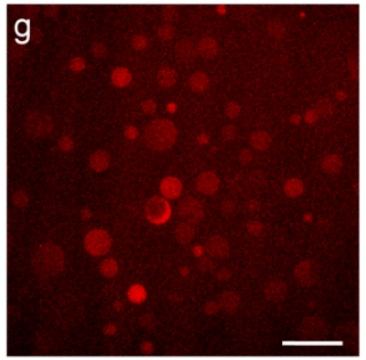

ferricyanide/ (Arg) with dye (PolyA)

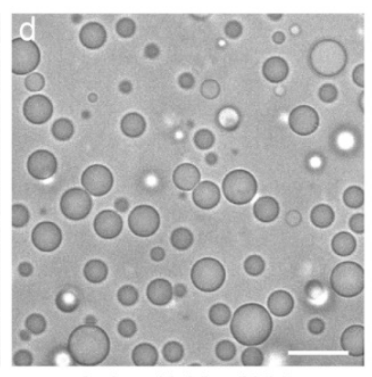

ferricyanide/ $\left(\operatorname{Lys}(\mathrm{Me})_{3}\right)_{30}$

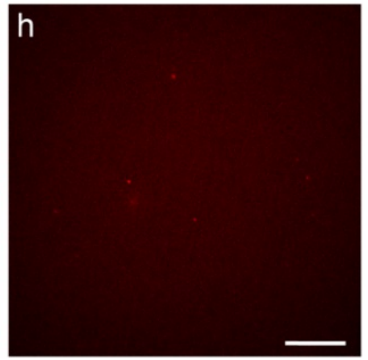

ferricyanide/ (Arg) with dye (Nile red)

Supplementary Figure 4. Optical microscope images of ferricyanide/(Lys) $)_{20}$ (a), ferricyanide/(Lys $\left.(\mathrm{Me})_{3}\right)_{20}$ (b), ferricyanide/(Lys) $)_{30}(\mathrm{c})$, ferricyanide/(Lys(Me) $\left.)_{3}\right)_{30}(\mathrm{~d})$, and ferricyanide/(Arg $)_{10}(\mathrm{e})$ coacervates. Fluorescence images of ferricyanide/(Arg) 10 coacervate droplets selectively uptake of NADH (f), Poly- $\mathrm{A}_{15}\left(\mathrm{Cy} 5-\mathrm{A}_{15}\right)$ (g), and Nile red (h).

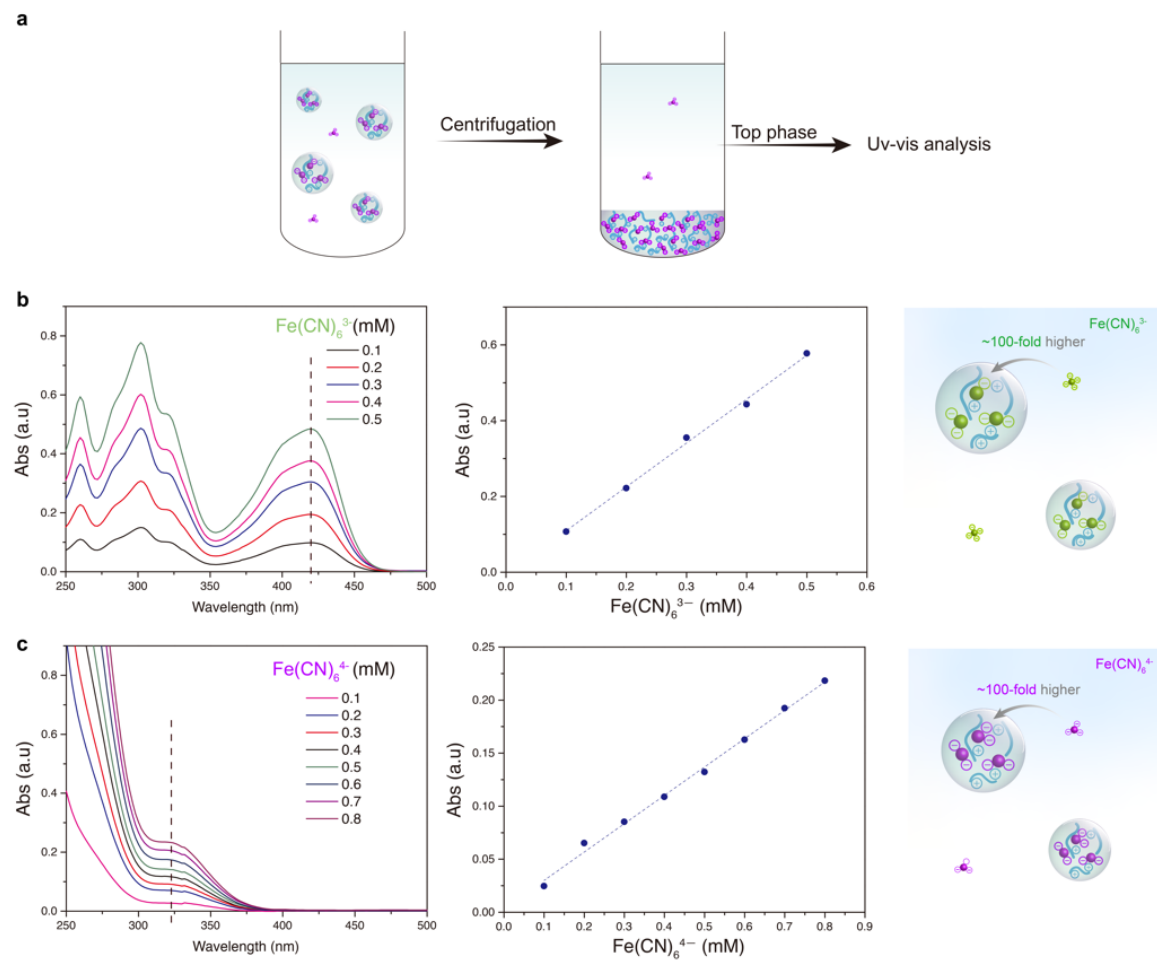

Supplementary Figure 5. (a) Scheme of the method to determine the ferricyanide concentrations inside and outside the droplet phase. (b, c) UV-vis spectra of the ferricyanide or ferrocyanide concentration in top phase at different concentrations, and the corresponding standard curve of the ferricyanide/ferrocyanide absorbance. We calculated the ferricyanide and ferrocyanide concentrations of the top solution from the standard curve, and then the ferricyanide and ferrocyanide concentrations inside the coacervate phase can be known. 

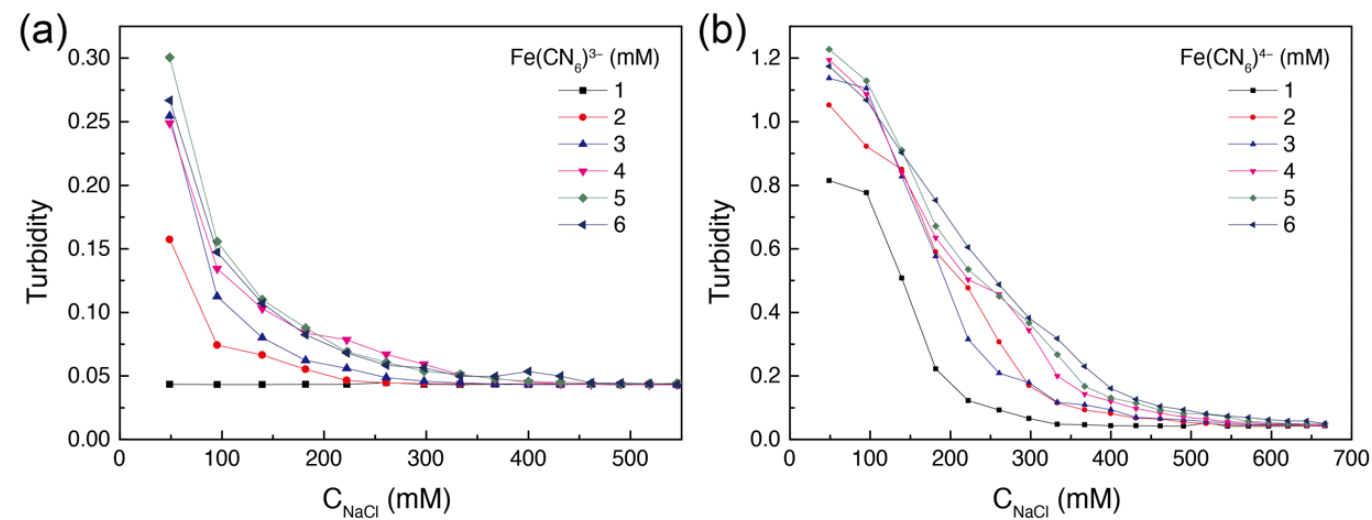

Supplementary Figure 6. Turbidity of $\mathrm{Fe}(\mathrm{CN})_{6}{ }^{3-} / \mathrm{Fe}(\mathrm{CN})_{6}{ }_{6}^{4-}$-pLys mixtures as a function of concentration of added $\mathrm{NaCl}$ ( $\mathrm{a}$ and $\mathrm{b}$ ). The mixtures contained a fixed concentration of $50 \mathrm{mM}$ Tris buffer, $5 \mathrm{mM}$ pLys (monomer units). The mixtures containing ferricyanide were titrated with $\mathrm{NaCl} 0.5 \mathrm{M}$, while the mixtures containing Ferrocyanide were titrated with $\mathrm{NaCl} 2 \mathrm{M}$.
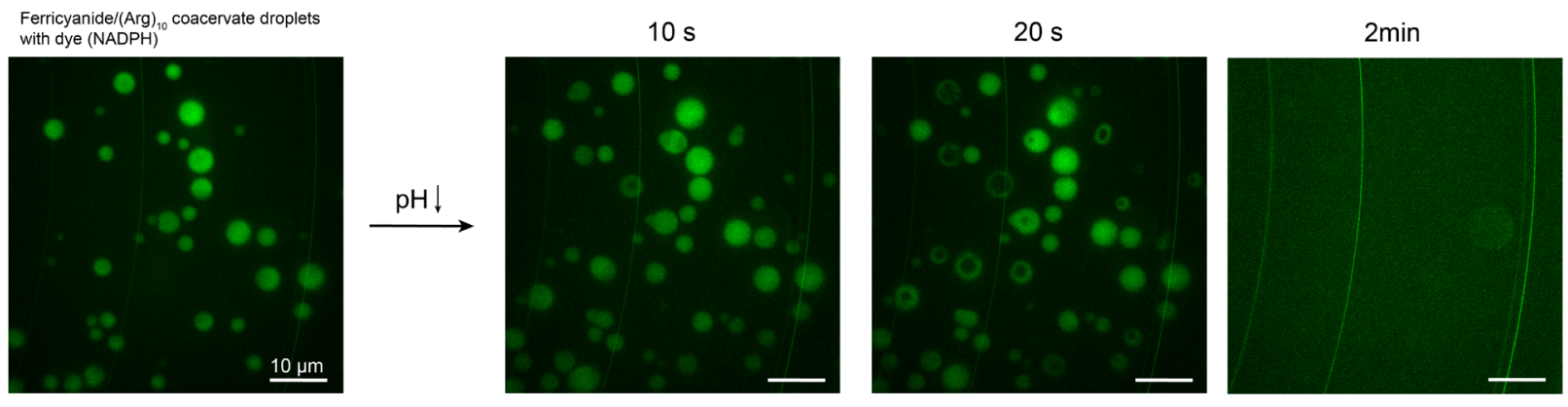

Supplementary Figure 7. Confocal micrographs of ferricyanide/(Arg) $)_{10}$ protocells (stained with NADH), time series of representative protocells after $\mathrm{pH}$-triggered redox reaction.
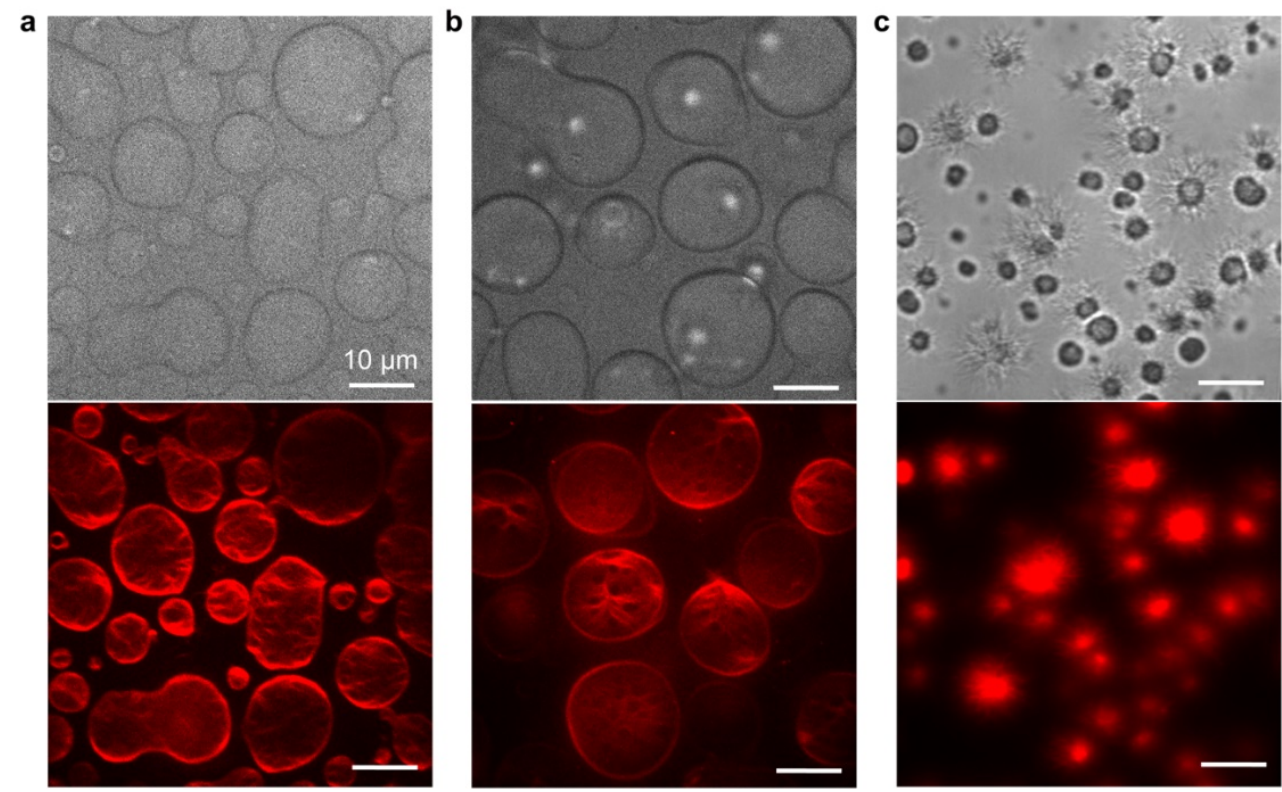

Supplementary Figure 8. Confocal micrographs of ferricyanide/(Lys(Me) $\left.)_{3}\right)_{20}(a)$, ferricyanide/(Lys $\left.(\mathrm{Me})_{3}\right)_{30}(\mathrm{~b})$, and ferricyanide/(Lys) $)_{30}$ (c) protocells (with dye, Nile red) after addition of BC. Scale bar, $10 \mu \mathrm{m}$. 


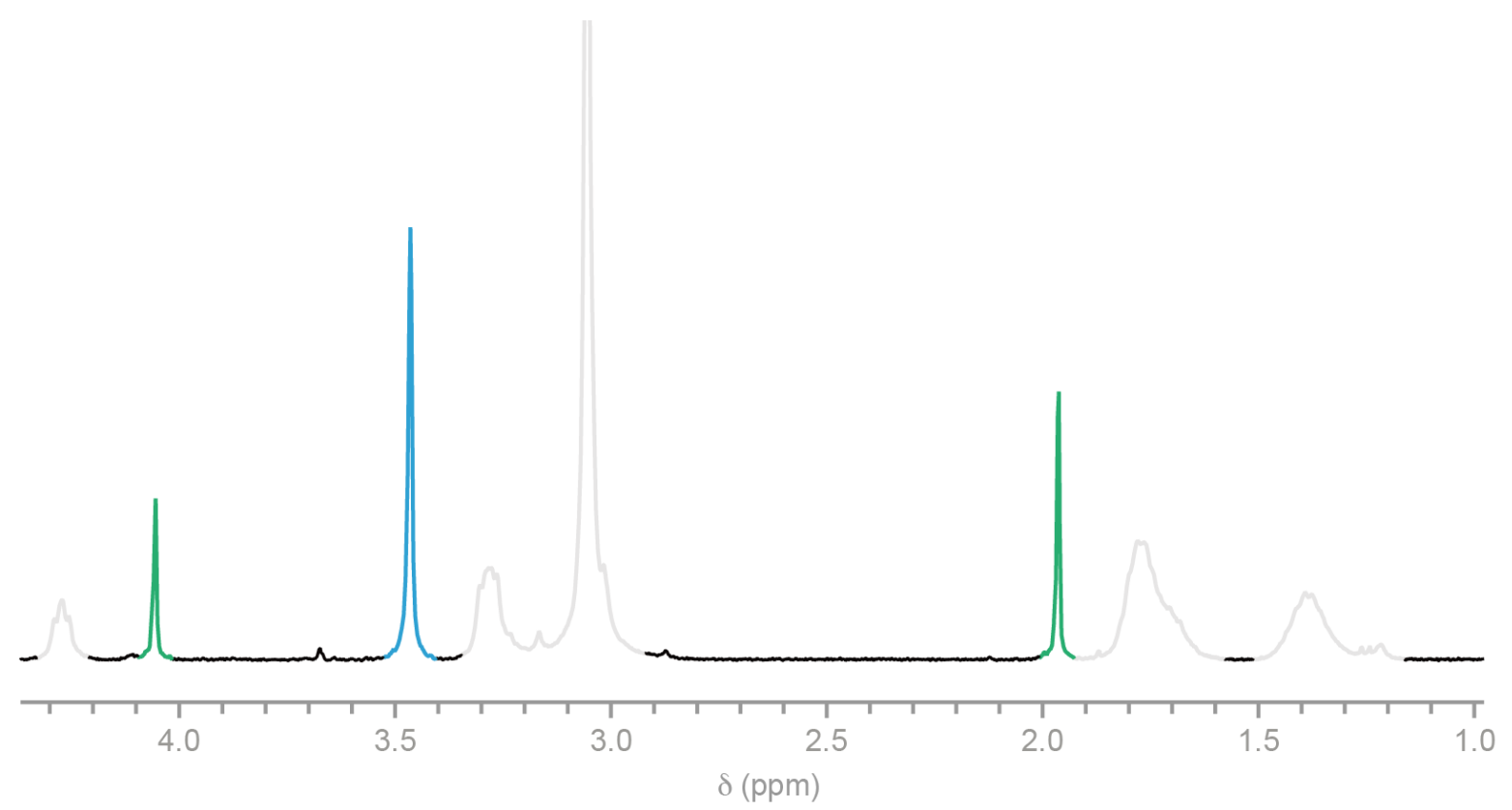

Supplementary Figure 9. ${ }^{1} \mathrm{H}$ NMR spectrum of peptide ligation reaction in control solution without ferricyanide, no ligation product observed after $3 \mathrm{~h}$. (Ac-Gly-SH (8mM, green), Gly (3 equiv., blue), (ferrocyanide, 6mM), $\left(\left(\operatorname{Lys}(\mathrm{Me})_{3}\right)_{30}\right.$, gray $\left.)\right)$.

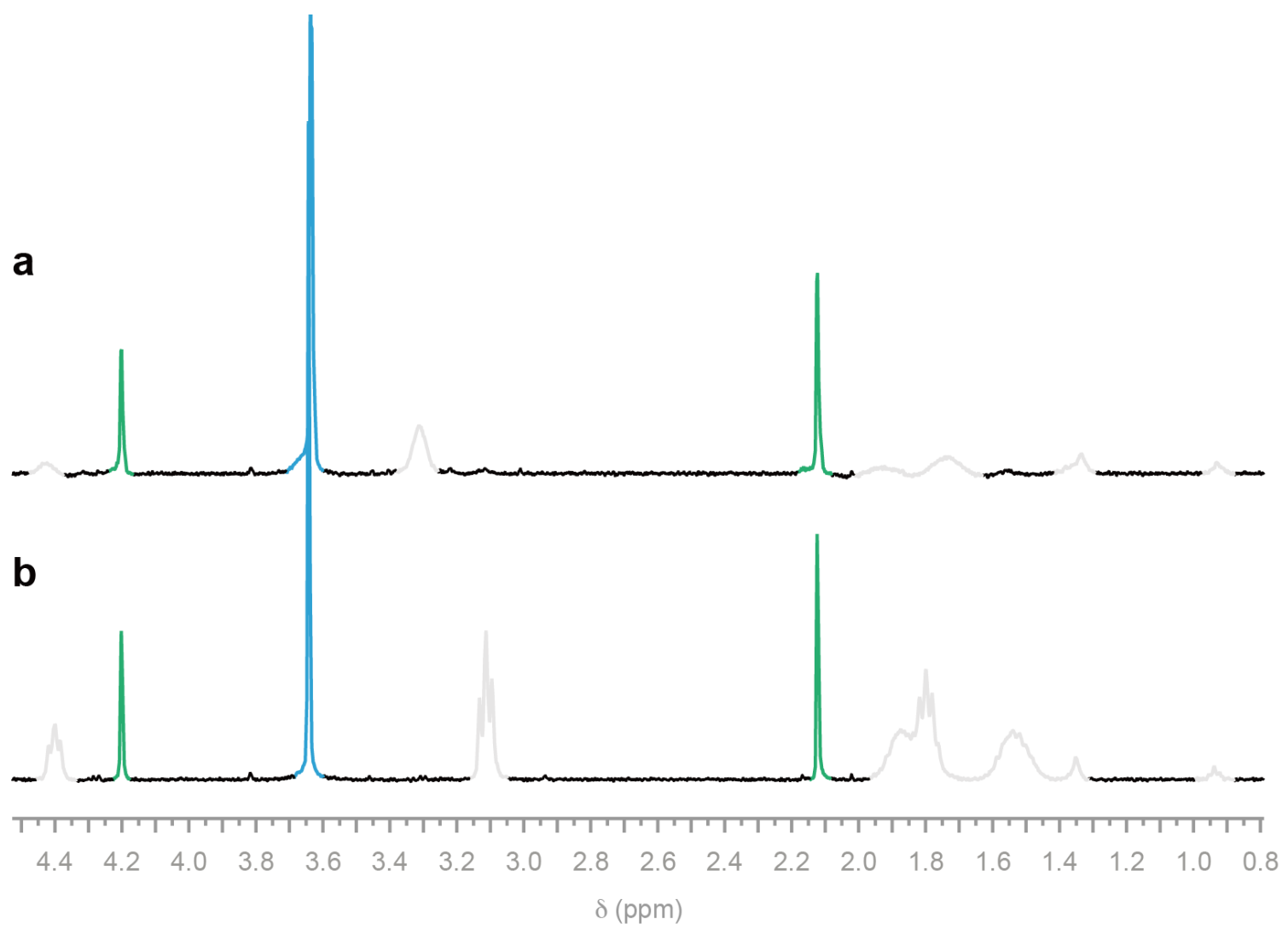

Supplementary Figure 10. ${ }^{1} \mathrm{H}$ NMR spectrum of peptide ligation reaction in ferrocyanide/(Arg) $)_{10}$ (a) and ferrocyanide/(Lys) ${ }_{20}$ protocells, no ligation product observed after $3 \mathrm{~h}$. (Ac-Gly-SH (8mM, green), Gly (3 equiv., blue), (ferrocyanide, $6 \mathrm{mM})$, (polycations, gray)). 


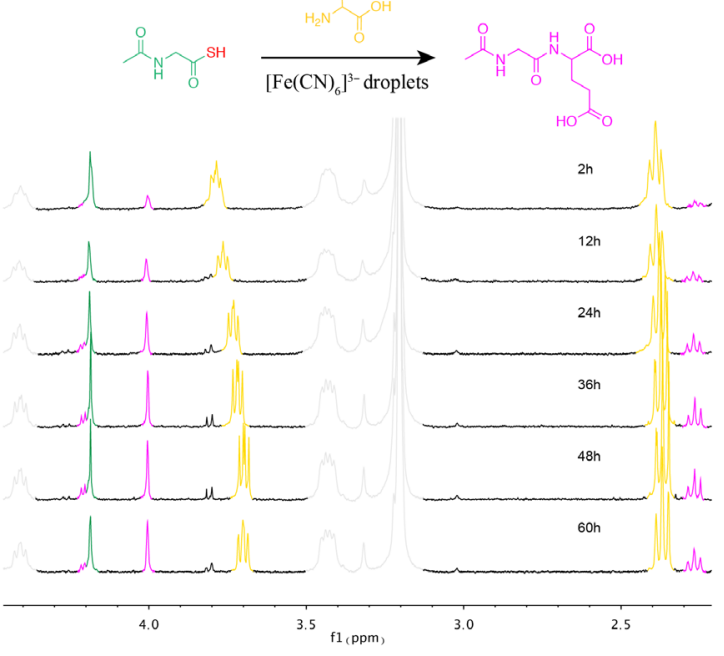

b

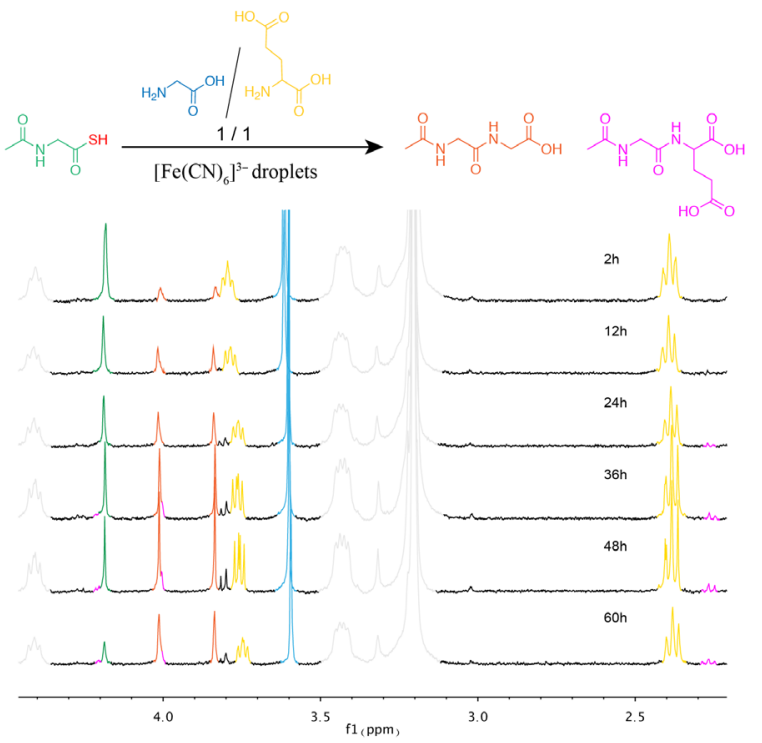

Supplementary Figure 11. (a) Time series of ${ }^{1} \mathrm{H}$ NMR spectrum of peptide ligation reaction in ferricyanide/(Lys $\left.(\mathrm{Me})_{3}\right)_{20}$ protocells. (Ac-Gly-SH $(8 \mathrm{mM}$, green), Glu $(24 \mathrm{mM}$, yellow), (ferricyanide, $8 \mathrm{mM})$, (polycations, gray)). (b) Time series of ${ }^{1} \mathrm{H}$ NMR spectrum of peptide ligation reaction in ferricyanide/(Lys(Me) $\left.)_{3}\right)_{20}$ protocells. (Ac-Gly-SH ( $8 \mathrm{mM}$, green), Glu (12 mM, yellow), Gly (12 mM, blue) (ferricyanide, $8 \mathrm{mM})$, (polycations, gray)).

Supplementary Table 1. Yields for the products of prebiotic oxidative coupling of a-aminoacetyl thioacid Ac-GlySH (8 mM) with amino acid mixture $\mathbf{A} \mathbf{A}_{1} / \mathbf{A A}_{2}$ (ratio 1:1, $\left.12 \mathrm{mM}: 12 \mathrm{mM}\right)$ and Ferricyanide $(8 \mathrm{mM}) /\left(\operatorname{Lys}(\mathrm{Me})_{3}\right)_{20}$ (Lys monomer $24 \mathrm{mM}$ ) coacervates dispersion, $\mathrm{pH} 9$, unless stated otherwise.

\begin{tabular}{|c|c|c|}
\hline \multirow{3}{*}{$\begin{array}{l}\text { Amino Acid mixture } \\
\qquad A A_{1}: A A_{2} 1: 1\end{array}$} & \multirow{2}{*}{\multicolumn{2}{|c|}{ 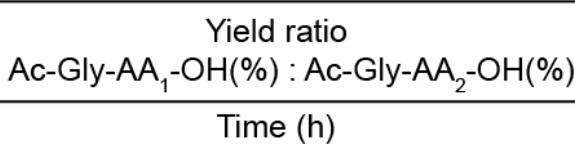 }} \\
\hline & & \\
\hline & $4 \mathrm{~h}$ & $60 \mathrm{~h}$ \\
\hline Gly:Glu & $10: 0$ & $10: 1$ \\
\hline Gly:Ala & $6: 1$ & $5: 1$ \\
\hline Gly:Phe & 4.8:1 & $3.5: 1$ \\
\hline Ala:Glu & $1.7: 1$ & $1.5: 1$ \\
\hline Glu:Phe & $3.5: 1$ & $1.7: 1$ \\
\hline
\end{tabular}




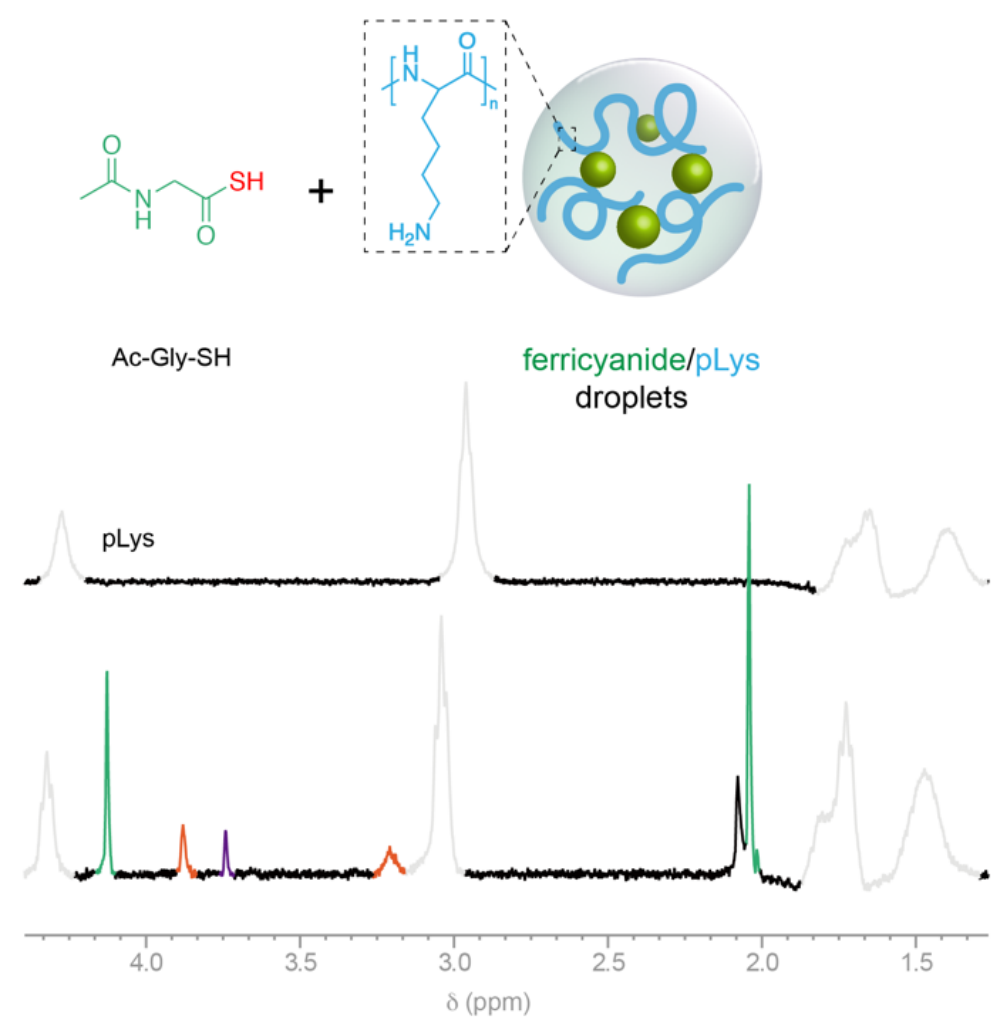

Supplementary Figure 12. ${ }^{1} \mathrm{H}$ NMR spectrum showing the thioacid ligation reaction of Ac-Gly-SH (8 $\mathrm{mM}$, green) with ferricyanide $(8 \mathrm{mM}) / \mathrm{pLys}\left(15-30 \mathrm{~K}\right.$, Lys monomer $24 \mathrm{mM}$, gray) coacervates to yield $\mathrm{pLys} \varepsilon-\mathrm{NH}_{2}$ ligation (orange).

\section{Supplementary references}

1. Okamoto, R.; Haraguchi, T.; Nomura, K.; Maki, Y.; Izumi, M.; Kajihara, Y., Regioselective alpha-Peptide Bond Formation Through the Oxidation of Amino Thioacids. Biochemistry 2019, 58 (12), 1672-1678.

2. Canavelli, P.; Islam, S.; Powner, M. W., Peptide ligation by chemoselective aminonitrile coupling in water. Nature 2019, 571 (7766), 546-549.

3. Granados, E. N.; Bello, J., Alkylated poly(amino acids). I. Conformational properties of poly(Ne-trimethyl- Llysine) and poly(N8-trimethyl-L-ornithine). Biopolymers 1976, 18, 1479-1486. 